\title{
Turbulence kinetic energy budget during the afternoon transition - Part 2: A simple TKE model
}

\author{
Erik Nilsson ${ }^{1,2}$, Marie Lothon ${ }^{1}$, Fabienne Lohou ${ }^{1}$, Eric Pardyjak ${ }^{3}$, Oscar Hartogensis ${ }^{4}$, and Clara Darbieu ${ }^{1}$ \\ ${ }^{1}$ Laboratoire d'Aerologie, University of Toulouse, CNRS, Toulouse, France \\ ${ }^{2}$ Department of Earth Sciences, Uppsala University, Uppsala, Sweden \\ ${ }^{3}$ Department of Mechanical Engineering, Utah University, Salt Lake City, UT, USA \\ ${ }^{4}$ Meteorology and Air Quality Section, Wageningen University, Wageningen, the Netherlands \\ Correspondence to: Erik Nilsson (erik.nilsson@met.uu.se, erik.nilsson@aero.obs-mip.fr)
}

Received: 11 September 2015 - Published in Atmos. Chem. Phys. Discuss.: 2 November 2015

Revised: 3 July 2016 - Accepted: 4 July 2016 - Published: 19 July 2016

\begin{abstract}
A simple model for turbulence kinetic energy (TKE) and the TKE budget is presented for sheared convective atmospheric conditions based on observations from the Boundary Layer Late Afternoon and Sunset Turbulence (BLLAST) field campaign. It is based on an idealized mixedlayer approximation and a simplified near-surface TKE budget. In this model, the TKE is dependent on four budget terms (turbulent dissipation rate, buoyancy production, shear production and vertical transport of TKE) and only requires measurements of three available inputs (near-surface buoyancy flux, boundary layer depth and wind speed at one height in the surface layer) to predict vertical profiles of TKE and TKE budget terms.

This simple model is shown to reproduce some of the observed variations between the different studied days in terms of near-surface TKE and its decay during the afternoon transition reasonably well. It is subsequently used to systematically study the effects of buoyancy and shear on TKE evolution using idealized constant and time-varying winds during the afternoon transition. From this, we conclude that many different TKE decay rates are possible under time-varying winds and that generalizing the decay with simple scaling laws for near-surface TKE of the form $t^{\alpha}$ may be questionable.

The model's errors result from the exclusion of processes such as elevated shear production and horizontal advection. The model also produces an overly rapid decay of shear production with height. However, the most influential budget terms governing near-surface TKE in the observed sheared convective boundary layers are included, while only second-
\end{abstract}

order factors are neglected. Comparison between modeled and averaged observed estimates of dissipation rate illustrates that the overall behavior of the model is often quite reasonable. Therefore, we use the model to discuss the lowturbulence conditions that form first in the upper parts of the boundary layer during the afternoon transition and are only apparent later near the surface. This occurs as a consequence of the continuous decrease in near-surface buoyancy flux during the afternoon transition. This region of weak afternoon turbulence is hypothesized to be a "pre-residual layer", which is important in determining the onset conditions for the weak sporadic turbulence that occur in the residual layer once nearsurface stratification has become stable.

\section{Introduction}

The daytime atmospheric boundary layer (ABL) is characterized by unstable stratification, turbulent mixing of momentum, heat, scalars and buoyancy-driven eddies. These large eddies are generated by a strong surface heat flux but are also influenced by wind shear (Stull, 1988). This is apparent near the surface as seen in our companion paper, Nilsson et al. (2016), which we will refer to as Part 1.

During the course of any day, atmospheric boundary layer turbulence will naturally respond to different levels of shear and buoyancy production, directly influencing the level of turbulence kinetic energy (TKE). In addition, transport and dissipation of TKE can change substantially from hour to hour as well as on shorter and longer timescales, thereby in- 
fluencing the level of TKE at specific heights in the ABL. Modeling the time evolution of the boundary layer for growth and decay phases of turbulence under unstable conditions can be a very challenging task, but it is important for many applications (e.g., dispersion of pollutants).

Several important earlier modeling studies of the daytime unstable ABL should be mentioned. The early work of Nieuwstadt and Brost (1986) and later studies of Pino et al. (2006) considered a very abrupt instantaneous shutdown of sensible heat flux to zero in large-eddy simulation (LES), which may best correspond to a modeling effort of unusual solar eclipse events. Sorbjan (1997) instead considered using a cosine-shaped surface heat flux forcing, which can fit measurements relatively well for the afternoon time period (Nadeau et al., 2011). In Sorbjan (1997), a forcing timescale implying a length of the afternoon period of only about $1.4 \mathrm{~h}$ was used, which can often be considered very short in midlatitudes. The study of van Driel and Jonker (2011) also performed idealized simulations for the unstable boundary layer using LES with a variety of non-stationary surface heat flux forcing functions and emphasized that mixed-layer modeling can be quite successful as long as the forcing timescales are not short in comparison to the eddy turnover timescale.

Goulart et al. (2003, 2010) studied TKE using a theoretical spectral model and LES data and showed a slower decay of TKE during the afternoon transition when including wind shear in their modeling attempts. This was also clearly shown for TKE averaged over the boundary layer depth in Pino et al. (2006). Beare et al. (2006) also studied afternoon and evening transition leading up to the early morning boundary layer using LES, and several studies (Brown et al., 2002; Kumar et al., 2006; Basu et al., 2008) also attempted to model a realistic diurnal cycle using LES. These studies did not, however, specifically address the representation of the evolution of TKE. Special attention was paid to the evolution of the turbulent kinetic energy during the Boundary Layer Late Afternoon and Sunset (BLLAST) field campaign. TKE was sampled by a combination of independent instruments, and this was a specificity and one of the aims of the field campaign (Lothon et al., 2014; Couvreux et al., 2016). Rizza et al. (2013) studied TKE evolution with LES and showed that boundary layer averaged TKE can obtain exponents of the decay power law $t^{\alpha}$ from at least -2 to -6 as previously shown for surface layer TKE (Nadeau et al., 2011) using measurements.

For the BLLAST field campaign, several LES studies (Blay-Carreras et al., 2014; Pietersen et al., 2015; Darbieu et al., 2015b) have been carried out on specific days of the field campaign. These studies have provided analysis of TKE evolution and turbulence structure (Darbieu et al., 2015b) and have taken into account external forcing effects such as for instance subsidence (Pietersen et al., 2015; Blay-Carreras et al., 2014) and influence of the residual layer from a previous day on the growth of the morning boundary layer (BlayCarreras et al., 2014). Model experiments for several days of the field campaign, rather than specific case studies, are also very beneficial for aiding in understanding the differences between days better. In the context of BLLAST, Couvreux et al. (2016) evaluated the ability of numerical weather prediction models to predict TKE evolution for all intensive observation period (IOP) days. These studies did not, however, cover the evolution of the TKE budget in the afternoon transition.

Nadeau et al. (2011) managed to rather successfully model near-surface TKE decay in the afternoon for very convective days using a simple heuristic TKE budget model. Their model's only inputs are boundary layer depth and buoyancy flux, and it uses a simple parametrization for dissipation of TKE. In Part 1, TKE budget calculations showed that a realistic modeling of near-surface TKE for the observational period during BLLAST requires accounting for shear production and vertical transport of TKE in addition to dissipation and buoyant production.

In this paper, we present a simple one-dimensional TKE budget model based on the analysis presented in Part 1 and assumptions about approximate height dependencies of TKE budget terms in the mixed layer. We use this model to carry out simulations for nine IOP days where near-surface measurements and TKE budget estimates for both morning and afternoon periods were available. In this way, we can compare our simulated TKE at different heights to observations and discuss directly how the estimated budget terms act in the model to underestimate or overestimate TKE at specific times. We want to stress that this model has been developed with the aim of aiding in the understanding of the most important processes that govern TKE evolution for sheared convective situations, but it should not be regarded as a complete description of the complex reality. As will be discussed further in the text, the model does not include processes such as elevated shear production and horizontal advection of TKE, which may be important at specific times. We use observations from several different land cover types to explore the sensitivity of the modeled boundary layer dissipation rates in relationship to those observed over the heterogeneous BLLAST field campaign landscape. This heterogeneity challenges some of our modeling assumptions. We insist on carrying out the study with a simple model for near-surface TKE and TKE budget terms because it is an important first step before more complexity and processes may be added. Compared to the model proposed in Nadeau et al. (2011), which required a prescribed boundary layer depth and near-surface buoyancy flux, we add a prescribed near-surface wind speed to carry out our modeling efforts.

The paper is structured as follows. Firstly, in Sect. 2 we introduce the model main goal and description. Here we describe the different parts of the simple TKE model and illustrate the height dependence of model terms. In Sect. 3, we guide the reader further to the relevant data sets used in the paper and further documentation about how the data were selected and treated for our modeling effort. This is followed 
in Sect. 4 by evaluation of near-surface TKE and TKE budget terms for nine simulated IOP days including discussion of potential sources of errors in TKE prediction. In Sect. 5, we explore modeled dissipation rate for the boundary layer, using observed fluxes and winds from different surface land covers and an area-averaged flux, in comparison to observed dissipation rate and discuss the formation of a "pre-residual layer" during the afternoon transition. In Sect. 6, we use the model to simulate near-surface TKE for a variety of idealized afternoon conditions and discuss the results in relationship to previously proposed "decay laws" of turbulence. Finally, we conclude and summarize in Sect. 7.

\section{Model main goal and description}

In this section, we describe our simple model for the atmospheric boundary and surface layer turbulence kinetic energy. From inputs of time series of near-surface buoyancy flux, wind speed at one height in the surface layer and boundary layer depth estimates the model predicts vertical profiles of terms in the TKE budget equation as well as TKE. The model is initialized in the morning transition and gives an approximate description of the surface and boundary layer evolution in terms of TKE and its budget terms during unstable conditions until the end of the afternoon. Observations for one BLLAST case (20 June) are shown as the model terms are introduced, even if the observations are described in more details in Sect. 3. A more extensive evaluation of near-surface TKE budget terms is given in Sect. 4 .

\subsection{The governing TKE equation}

In this work, we consider a simplified budget for TKE of the following form, assuming no advection and horizontal homogeneity:

$$
\begin{aligned}
& \underbrace{\frac{\partial E}{\partial t}}_{\text {Tendency }}=\underbrace{-\overline{u^{\prime} w^{\prime}} \frac{\partial U}{\partial z}}_{\text {Shear production: } S}+\underbrace{\frac{g}{\bar{\theta}} \overline{w^{\prime} \theta_{v}^{\prime}}}_{\text {Buoyancy production: } B}- \\
& \underbrace{-\frac{\partial \overline{w^{\prime} E^{\prime}}}{\partial z}-\frac{\partial \overline{w^{\prime} p^{\prime} / \rho_{0}}}{\partial z}}_{\text {Transport: } T} \underbrace{-\epsilon}_{\text {Dissipation: } D} .
\end{aligned}
$$

Here, TKE $(=E)$ denotes $\frac{1}{2}\left(\overline{u^{\prime 2}}+\overline{v^{\prime 2}}+\overline{w^{\prime 2}}\right)$, where $u^{\prime}$, $v^{\prime}$ and $w^{\prime}$ are respectively the instantaneous deviations of along-wind, cross-wind, and vertical wind components from their respective mean values. $U$ is the magnitude of the mean wind, which varies with height, $z ; g$ is the acceleration of gravity; $\bar{\theta}$ is mean absolute temperature; $\theta_{\mathrm{v}}^{\prime}$ is the instantaneous deviation of virtual potential temperature from its mean value; $\rho_{0}$ is the air density; $p^{\prime}$ is the instantaneous deviation of air pressure; and $\epsilon$ is the mean dissipation rate of TKE.
The physical interpretation of the five terms in Eq. (1), from left to right, is local time rate of change of TKE, shear production of TKE, buoyancy production of TKE, vertical divergence of the total transport of TKE, and dissipation rate of TKE.

Given simple parametrization for the right-hand-side terms of Eq. (1) and specified initial profile of the TKE, the budget equation can be used to solve for the evolution of turbulence kinetic energy $E(t)$. Our choice of using a simple parametrization of budget terms instead of first-order closure stems from us originally aiming at a simple surface layer parametrization, which still takes into account of simplified mixed-layer effects. The eddy-diffusivity concept can be another effective approach for single-column modeling although typical assumptions such as for instance relating transport of TKE to the gradient of TKE can be questionable as shown in Puhales et al. (2013). Below we describe the simple modeling of surface fluxes, the assumed flux gradient relationship, treatment of the height dependence of the various budget terms and the initial conditions. We also compare our model to the simple TKE budget model from Lenschow (1974) in Sect. 2.4.

\subsection{Treatment of surface fluxes, flux gradient relationship}

An important driving force for the atmospheric boundary layer turbulence in unstable conditions is the surface buoyancy flux, which controls near-surface buoyancy production in this simple model. In first instance, we will prescribe these, as determined in Part 1, from observations made at $3.23 \mathrm{~m}$ from the ground (see later in the text for more details). In Sect. 5, we will go on to use the preprocessed available time series of sensible heat flux (SH), latent heat flux $\left(L_{\mathrm{v}} E\right)$ and observed potential temperature time series $\bar{\theta}$ from the other surfaces. The following relationship is then used assuming there is no influence from liquid water flux:

$B_{0}=\frac{g}{\rho c_{p} \bar{\theta}}\left(S H+\left(0.61 c_{p} \bar{\theta} / L_{\mathrm{v}}\right) L_{\mathrm{v}} E\right)$.

Here, $B_{0}$ is the buoyancy production term used at the first grid point above the surface (or above the displacement height $d$ in the case of the forest), $c_{p}$ is the specific heat capacity of air, and $L_{\mathrm{v}}$ is the specific latent heat of vaporization.

In Part 1, shear production was shown to be an important source of turbulence production, especially near the surface. To model shear production, we use an idealized MoninObukhov similarity-based flux gradient relationship (Wilson, 2001) to determine the vertical gradient of mean wind speed strictly applicable to a locally homogeneous quasi-steady atmospheric surface layer:

$\frac{\partial U}{\partial z}=\frac{u_{*}}{k z} \phi_{m}\left(\frac{z}{L}\right)$.

Here, $u_{*}$ is friction velocity, $k$ the von Kármán constant (set to 0.4) and $L$ is the Obukhov length scale ( $L=$ 
$\left.-\frac{\bar{\theta} u_{*}^{3}}{k g\left(\overline{w^{\prime} \theta_{\mathrm{v}}^{\prime}}\right)_{0}}\right)$, in which $\left(\overline{w^{\prime} \theta_{\mathrm{v}}^{\prime}}\right)_{0}$ is the kinematic virtual temperature flux at surface. Based on fits to extensive data from Högström (1988), Wilson (2001) proposed the following functional form for the non-dimensional wind gradient, $\phi_{\mathrm{m}}$ :

$\phi_{m}=\left(1+3.6|z / L|^{2 / 3}\right)^{-1 / 2}$,

which for unstable conditions integrates to the relatively simple mean wind profile

$U(z)=\frac{u_{*}}{k}\left[\ln \frac{z}{z_{0}}-3 \ln \left(\frac{1+\sqrt{1+3.6|z / L|^{2 / 3}}}{1+\sqrt{1+3.6\left|z_{0} / L\right|^{2 / 3}}}\right)\right]$.

It should be noted here that a different functional form for the non-dimensional wind gradient was found in Part 1, but here we chose to keep the consensus value of von Kármán's constant. We consider it may be other non-dimensional parameters than $z / L$ that is also needed to improve shear production estimates. As we shall see in Sect. 4 the chosen functional form provides a reasonable wind gradient and shear production very near the surface, but it is less good at increasing height.

To use the above wind speed relationship, we need to determine a $u_{*}$ value which also enters into the Obukhov length $L$. To do this, the wind speed is first extrapolated from the measurement height $z_{\mathrm{m}}$ to $z=10 \mathrm{~m}$ using

$U_{10}=U_{m} \frac{\ln \left(10 / z_{0}\right)}{\ln \left(z_{m} / z_{0}\right)}$

A simple drag coefficient or CD-curve approach $(\mathrm{CD}=$ $\left.u_{*}^{2} / U_{10}^{2}\right)$ is then used to form an initial estimate of $u_{*}$ with the following relationship (determined from measurements, see Fig. 1):

$\mathrm{CD}=A_{\mathrm{CD}} U_{10}+B_{\mathrm{CD}}$

where the empirical coefficients were computed to be $A_{\mathrm{CD}}=$ $2 \times 10^{-3} \mathrm{~s} \mathrm{~m}^{-1}$ and $B_{\mathrm{CD}}=5 \times 10^{-3}$ (unitless).

Using such a $u_{*}$ value directly in Eq. (5) would, however, not produce a wind speed that is consistent with the measured mean wind speed at height $z_{\mathrm{m}}$. Therefore, an iterative approach is used to determine a stability-corrected $u_{*}$ value in the following way. Firstly, a value for Obukhov length is calculated. Then, Eq. (5) is rewritten to solve for $u_{*}$ taking into account the influence of stability:

$u_{*}=$

$\left(k U\left(z_{m}\right)\right) /\left[\ln \frac{z_{m}}{z_{0}}-3 \ln \left(\frac{1+\sqrt{1+3.6\left|z_{m} / L\right|^{2 / 3}}}{1+\sqrt{1+3.6\left|z_{0} / L\right|^{2 / 3}}}\right).\right]$

This new $u_{*}$ value is used to calculate a new Obukhov length and the process is repeated 10 times so that converged $u_{*}$ and $L$ values are reached. Usually only two or three iterations are needed for sufficient convergence.

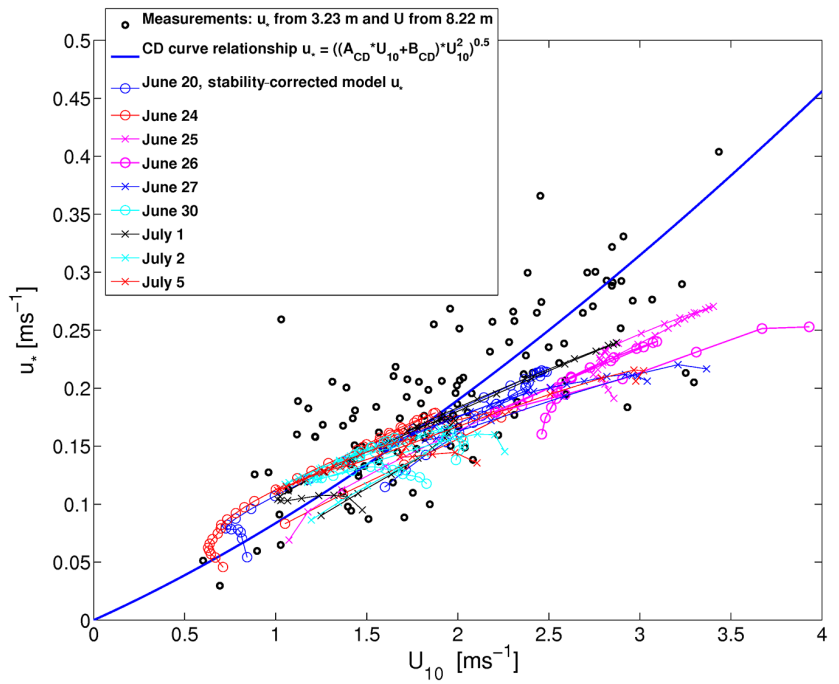

Figure 1. Measured hourly averaged $u_{*}$ from the $3.23 \mathrm{~m}$ level is shown as a function of wind speed at $8.22 \mathrm{~m}$ in black circles. Here all measurements in unstable conditions for the studied IOP days are shown. The model $u_{*}$ prediction from a simple CD curve relationship with $A_{\mathrm{CD}}=2 \times 10^{-3} \mathrm{~s} \mathrm{~m}^{-1}$ and $B_{\mathrm{CD}}=5 \times 10^{-3}$ (unitless) is shown as a blue line. The model stability-corrected $u_{*}$ values for nine IOP days are also shown in different colored lines with circles or crosses as a function of the $10 \mathrm{~m}$ model wind speed for every 20 min during the simulations.

In Fig. 1, the measured and modeled $u_{*}$ values are plotted as a function of wind speed. Measurements shown here come from a set of IOP days during the BLLAST field experiment, at 3.23 and $8.22 \mathrm{~m}$ above ground. Some variability is missed with this approach and it may lead to systematic underestimation in the modeled $u_{*}$ when winds are higher than $2 \mathrm{~m} \mathrm{~s}^{-1}$. As will be evident from time series presented in Sect. 4, some of the high values of measured $u_{*}$ at $3.23 \mathrm{~m}$ are, however, occurring very temporarily and are not always clearly linked to the mean wind at 8 or $10 \mathrm{~m}$. Hence, they are likely not being well predicted by the relationship formed from the input of one mean wind at one height.

\subsection{Height variation in modeled TKE budget terms}

Here, we describe the vertical height dependence that is assumed for each of the right-hand-side budget terms of Eq. (1). At the same time, we will briefly discuss the behavior of the corresponding measurements from the "divergence site" tower at four times during the afternoon of 20 June.

\subsubsection{Height dependence of the buoyancy term}

To describe the height variation in the boundary layer, we use idealized linear profiles of buoyant production. These profiles are in general agreement to the proposed shapes (based on measurements) from Lenschow et al. (1980) and the model of Lenschow (1974) but some differences are dis- 

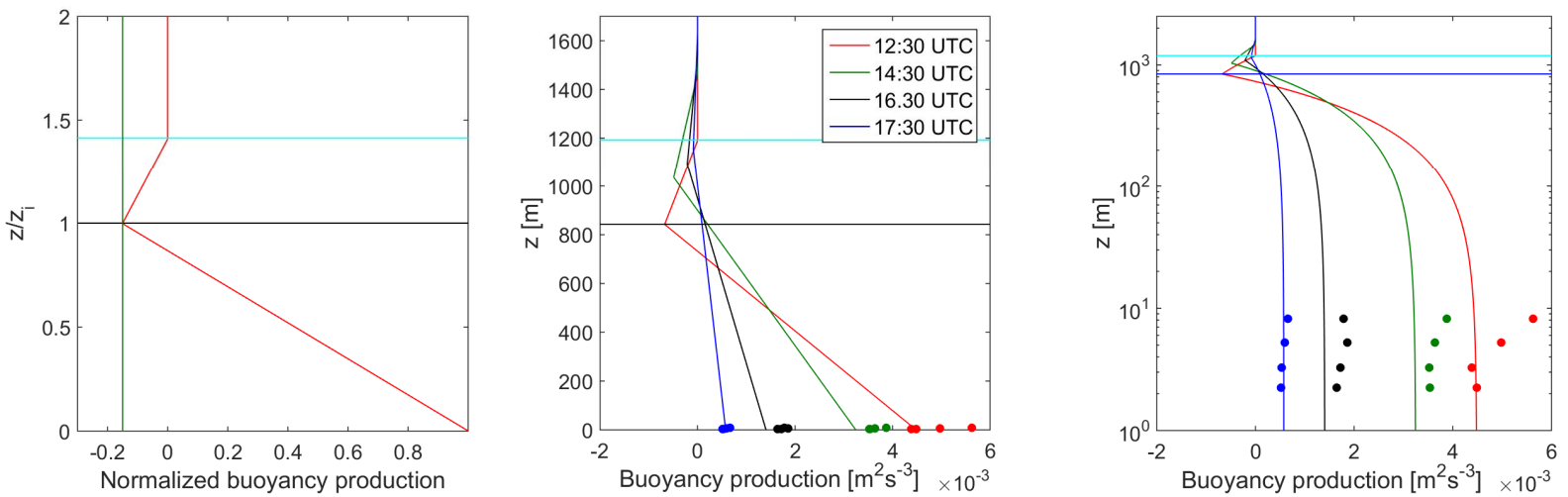

Figure 2. The left panel shows the simple modeled height dependence for the buoyancy production term in the TKE budget normalized by the surface value (in red). A vertical green line is added at -0.15 , which is the entrainment parameter value. A horizontal black line indicates the boundary layer depth $z_{i}$ defined from smoothed lidar measurements, whereas the horizontal light-blue line indicates $z_{i 0}$, the height of no turbulence. The middle panel shows an example of the evolution of the vertical profiles during the afternoon of 20 June. Measurements corresponding to the hourly averaged budget results for the four heights on the small tower are shown as colored dots. Boundary layer height $z_{i}$ and height of no turbulence $z_{i 0}$ for 12:30 UTC are shown as horizontal black and light-blue line. The right panel corresponds to the middle panel but shows the model and data on a semi-logarithmic scale to better display the near-surface behavior.

cussed in Sect. 2.4. We set an entrainment parameter $B_{\mathrm{E}}$ to -0.15 by analysis of the LES results for 20 June from Darbieu et al. (2015b). In Fig. 2, the normalized profile of buoyant production in the model is shown in the left panel. A linear decay is also assumed from $z_{i}$ up to a height of no turbulence $z_{i 0}$, which is defined from the vertical transport of TKE in Sect. 2.3.3.

In the middle and right panels of Fig. 2, we show the profiles of modeled buoyant production at four times during the afternoon transition. Near-surface hourly budget estimates centered on the corresponding times are also included. Boundary layer depth $z_{i}$ is prescribed from observed smoothed lidar measurements and hence evolve in time, but for clarity only $z_{i}$ and $z_{i 0}$ at 12:30 UTC are included as black and light-blue horizontal lines. It is clear that at 12:30 and 14:30 UTC the two upper measurement levels show higher values of buoyant production than the model. It is possible that some influence of large-scale submeso- or mesoscale fluctuations are causing higher values of fluxes at these heights in convective conditions. It is, however, unclear whether such features should be considered turbulence. We ignore some of these higher values, which is, as will be shown later, also not as consistent in time as the $3.23 \mathrm{~m}$ level measurements. It is, however, important to remember when interpreting these results that transport is calculated as a residual from other budget terms as described in Sect. 2.2.5 of Part 1.

\subsubsection{Height dependence of the shear production term}

The shear production considered in this simple model is given by $-\overline{u^{\prime} w^{\prime}(z)} \frac{\partial U}{\partial z}$, where the wind gradient is given by the expression discussed in Sect. 2.2. For the profile of stress, we first form a surface value $\overline{u^{\prime} w^{\prime}} 0=-u_{*}^{2}$ and then a lin- ear decay of the stress profile with height is assumed. More specifically, we assume that it decays to a value of 0 at $z_{i 0}$, where there is no turbulence and hence no stresses. Assuming linear stress profiles may be very reasonable for very convective conditions (Deardorff, 1972; Wyngaard, 2010) when their curvature is decreased to nearly zero and the contribution of $\overline{v^{\prime} w^{\prime}}$ stresses may also be smaller (Wyngaard, 2010). In more neutral conditions these assumptions may be more questionable, but keeping with our aim to formulate a simple model, we keep the assumption of linear stress profiles for all stability conditions. Also, Verkaik and Holtslag (2007) observed nearly linearly decaying momentum flux in slightly unstable conditions from several wind sectors and increasing momentum flux divergence with increasing stability in data from the Cabauw tower in the Netherlands.

We multiply our stress profile by the wind gradient expression (given by Eq. 3) to calculate the shear production term $S$ at each height. Very close to the surface, any shear production term that involves a logarithmic wind dependence will form a very high value. To address this, we replaced our first grid point value at $1 \mathrm{~m}$ above the surface (or first grid point above displacement height in the forest case) with a linear extrapolation of the second and third model level values.

In Fig. 3, we show the modeled and observed shear production for the afternoon of 20 June. It is clear that even though the model has roughly the correct order of magnitude at 2.23 and $3.23 \mathrm{~m}$, the modeled shear production term decays too quickly with height to a value of near zero at about $60 \mathrm{~m}$. The measured shear production may instead indicate that, in the middle of the boundary layer, some mixed-layer shear production takes place, which is not accounted for in this model driven by only surface measurements and boundary layer depth $z_{i}$. The wind gradient expression we have 

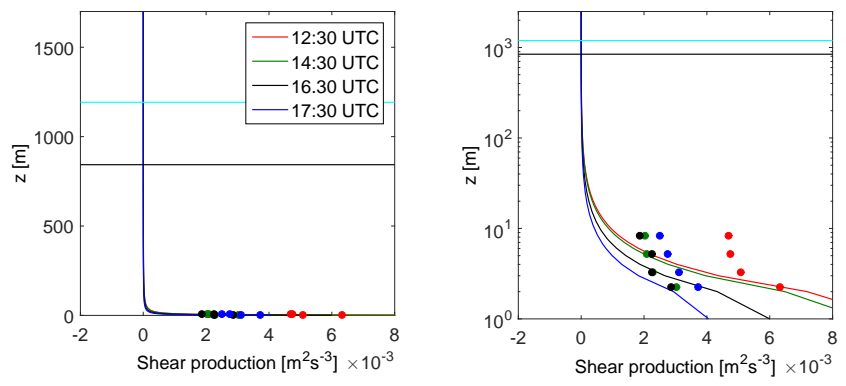

Figure 3. Left panel: modeled (in lines) and observed (in dots) shear production for the afternoon of 20 June. Red, green, black and blue correspond to 12:30, 14:30, 16:30 and 17:30 UTC, respectively. Horizontal black lines correspond to boundary layer depth $z_{i}$ and the light-blue horizontal line to height of no turbulence $z_{i 0}$ at 12:30 UTC. Right panel: the model and data in a semi-logarithmic representation to better display the near-surface behavior.
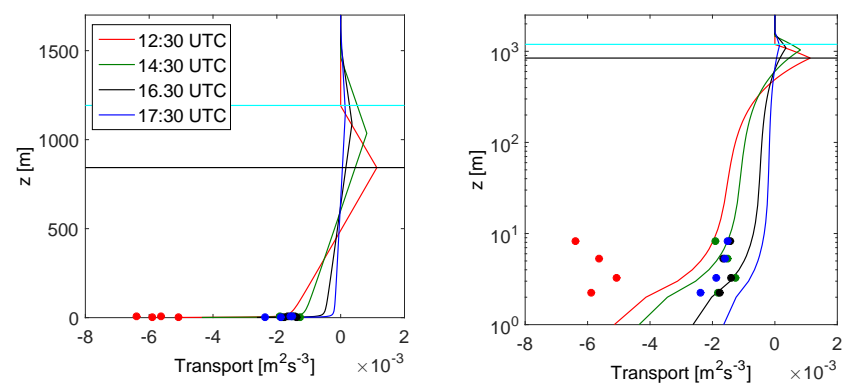

Figure 4. Same as Fig. 3 but for the transport term of the TKE budget.

used is also only meant to be used in the surface layer, and our measurements may suggest that it works best only in the limit of low heights.

\subsubsection{Height dependence of transport of TKE}

Figure 4 shows the modeled and observed transport term of the TKE budget. The total vertical transport consists of both pressure transport and turbulent transport (Stull, 1988), which often can be of different sign (Moeng and Sullivan, 1994). Here we will model only the sum of these two terms related to the available buoyancy and shear production of TKE. This is of course only an approximation of a more complex reality, but it is shown in Puhales et al. (2013) that more advanced eddy-diffusivity closures using the vertical gradient of TKE can also be questionable in comparison to LES data.

The modeled transport term consists of transport due to both buoyancy-produced TKE and shear-produced TKE. Such an approach may of course be criticized as turbulence in reality cannot be separated in such a way, but we are nevertheless not the first (Mangia et al., 2000) to suggest such an approach when attempting to simplify the situation for a simple model. Based on studying vertical profiles of trans- port in sheared convective LES (Moeng and Sullivan, 1994; Darbieu et al., 2015b), we adapt a very idealized transport term $T$ which consists of one part $T_{\mathrm{b}}$ more directly related to the buoyant production and one part transport $T_{\mathrm{S}}$ that is related to the shear production term. At each height they are related as

$T=T_{\mathrm{b}}+T_{\mathrm{s}}$.

The term $T_{\mathrm{b}}$ is given by a linear increase with height with slope $k_{1}$ for heights $z$ up to the boundary layer depth $z_{i}$ as

$T_{\mathrm{b}}(z)=T_{\mathrm{b}_{0}}+k_{1} z$.

At the boundary layer height $z_{i}$, the term reaches a maximum value $T_{\mathrm{b}_{\max }}$ and above the boundary layer depth a symmetric $-k_{1}$ slope is assumed so that the $T_{\mathrm{b}}$ is given by

$T_{\mathrm{b}}(z)=T_{\mathrm{b}_{\max }}-k_{1}\left(z-z_{i}\right)$.

This also determines the height of no turbulence $z_{i 0}$ as the height above $z_{i}$ where $T_{\mathrm{b}}(z)$ becomes 0 . The surface value $T_{\mathrm{b}_{0}}$ needs to be specified and it is determined by a fraction $T_{\mathrm{f}}$ of the total transport to the total near-surface production and the time-dependent surface buoyant production of TKE as

$T_{\mathrm{b}_{0}}(t)=-T_{\mathrm{f}} B_{0}(t)$.

We shall soon determine $T_{\mathrm{f}}$ from measurements, but first to solve Eqs. (10) and (11) we also need the slope $k_{1}$ which is given by

$k_{1}=\frac{T_{\mathrm{b}_{\max }}-T_{\mathrm{b}_{0}}}{z_{i}-0}$.

We solve for $T_{\mathrm{b}_{\max }}$ by requiring that the transport term $T_{\mathrm{b}}$ integrate to zero over the depth of the turbulent boundary layer. That is, from the surface to $z_{i 0}$. Given the transport fraction $T_{\mathrm{f}}$, the term $T_{\mathrm{b}}$ can now be obtained, but this only makes up one part of the total transport term in the model. In accordance with Eq. (9), we will first describe the transport related to shear production $T_{\mathrm{s}}$. This term is given by the expression

$T_{\mathrm{S}}(z, t)=-\left(T_{\mathrm{f}}-p\right) S(z, t)\left(1-z / z_{i 0}\right)$.

$S(z, t)$ is the height- and time-dependent shear production, $T_{\mathrm{f}}$ is the near-surface transport fraction and $p$ is a small positive free parameter that is determined such that the transport term $T_{\mathrm{s}}$ integrates to zero over the depth of the turbulent boundary layer. This produces a transport profile with a negative layer near the surface transporting some of the nearsurface shear-generated TKE to the upper parts of the boundary layer and a positive layer above indicating the transport term as a source of TKE at these heights. It also implies that the factor $p$ alters the near-surface transport fraction value, but usually only a few percent. 

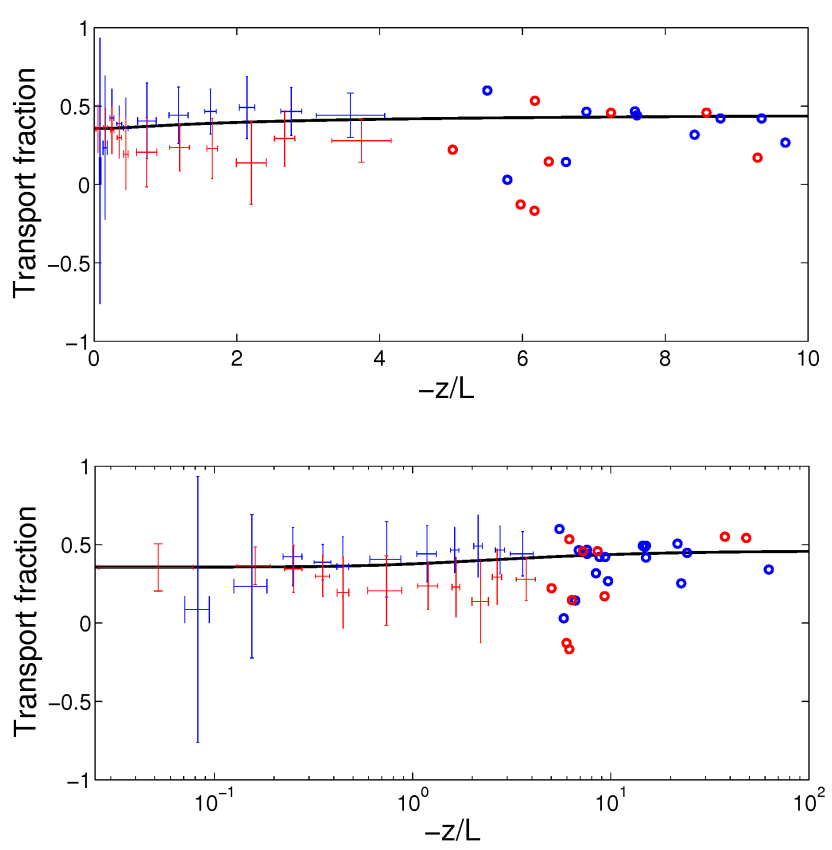

Figure 5. Near-surface transport fraction defined as minus the ratio of the total transport term to the sum of buoyancy and shear production. Red circles show hourly averaged data from the afternoon period from 12:00 UTC to zero buoyancy flux for $-z / L>5$ when data are sparse. Blue circles show the corresponding data from the morning period from positive sensible heat flux up until 12:00 UTC. The data coverage is successively better for more neutral stabilities and we show averaged data with error bars corresponding to \pm 1 standard deviation for both afternoon and morning period data for different intervals of the stability parameter $-z / L$. The intervals used have sizes of $0.1 z / L$ for $-z / L<1.0,0.5 z / L$ for $1<-z / L<3$ and one additional interval $3<-z / L<5$ is also included. The lower panel corresponds to the upper, but with a logarithmic abscissa to better display the very near neutral data and to also include a few hours with $-z / L$ values above 10 . In both panels, the model expression for transport fraction, which is applied at the first grid point ( $1 \mathrm{~m}$ above the surface), is included as a black line.

Finally, the transport fraction $T_{\mathrm{f}}$, defined as the ratio of (minus) the total near-surface transport and the sum of nearsurface shear and buoyancy production, is given as a $z / L$ dependent function, based on our TKE budget analysis in Part 1 where we determined $\phi_{T}$,

$T_{f}(z / L)=$

$\frac{-\phi_{T}}{\phi_{b}+\phi_{m}}=1+\frac{0.54 z / L-0.45}{0.7(1-15 z / L)^{-1 / 4}-z / L}$.

Equation (15) is compared to measurements in unstable conditions in Fig. 5, both for the afternoon and morning period with 4 orders of magnitude of variation in terms of the stability parameter $z / L$. It is a good match to data for the morning period (blue circles and bin-averaged data with error bars) except possibly very close to neutral where the error
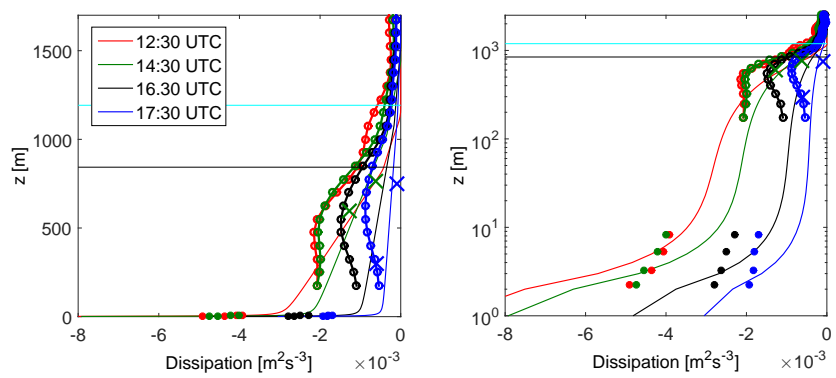

Figure 6. Same as Fig. 3 but for the dissipation term of the TKE budget. Here, smoothed and hourly averaged dissipation estimates are also included from the UHF wind profiler from $175 \mathrm{~m}$ (lines with circles). Estimates of dissipation from the Piper Aztec aircraft are included as colored crosses. Here, all aircraft flight legs during $1 \mathrm{~h}$ centered on 14:30 and 17:30 UTC, respectively, were averaged for height intervals of $75 \mathrm{~m}$.

bars are much larger mainly because of a specific time period in the morning of 25 June with indication of transport to the near-surface layers either from above or through a horizontal advection of TKE. Also, in comparison to the afternoon data, the expression is a good match very near neutral, but it potentially overestimates transport over the range of $-z / L$ between about 0.5 and 7 . It is, however, within the one standard deviation error bars. A different $z / L$ expression, which may fit the afternoon data better, would still not be general as it would degrade the performance during the morning period. Changing the near-surface transport fraction value to a constant of about 0.4 would also be possible and will only affect our simulation results slightly. Future work should be aimed at understanding whether the observed difference between the build-up and the decay phases of turbulence are linked to some other non-dimensional parameter combination. As a first approximation, we apply the presented relationship at the first grid point $1 \mathrm{~m}$ above the surface in our model. In the case of the forest, the relationship is instead applied at the first grid point above displacement height $d$.

The profile of total transport is shown in Fig. 4. A couple of things can be pointed out about the modeled profile. Due to the symmetric assumptions the ratio of $z_{i 0} / z_{i}$ is constant and equal to $\sqrt{2}$ except for a minor adjustment related to using fixed height levels in the model. The depth of the entrainment zone is also constant and about $38.5 \%$ of the boundary layer depth $\left(z_{i 0}\right)$ for our choice of an entrainment parameter of -0.15 taken from LES (Darbieu et al., 2015b). The value of $T_{\mathrm{b}_{\max }}$ will depend on $B_{0}(t)$ and the specified near-surface transport fraction $T_{\mathrm{f}}$, which, however, can be approximated as about 0.4 . The relationship for $T_{\mathrm{b}_{\max }}$ becomes roughly $T_{\mathrm{b}_{\max }}=(0.4 / \sqrt{2}) B_{0}$, which corresponds to approximately $28 \%$ of the near-surface buoyancy production value. This value will also dominate the maximum value in the total transport profile because the transport due to shear produc- 

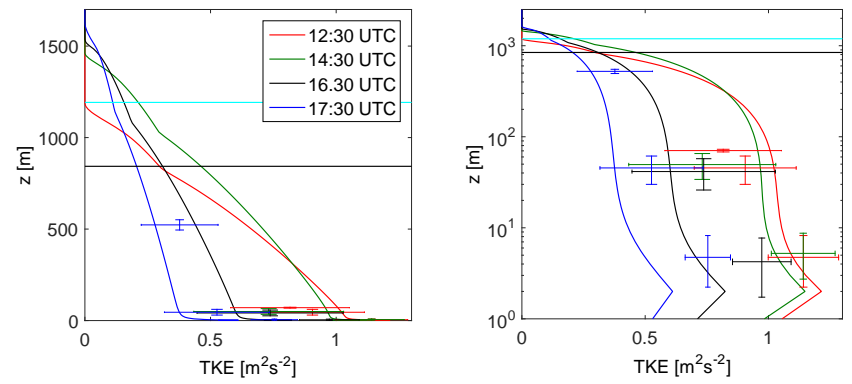

Figure 7. Profiles of TKE during the afternoon period on 20 June. The color scheme is the same as in earlier figures. The measurements on both the small tower $(2-8 \mathrm{~m})$ and the taller $60 \mathrm{~m}$ tower (30-60 m) were averaged and are shown with \pm 1 standard deviation. The data at times 14:30 and 16:30 UTC were displaced slightly in height to more easily see all error bars. The vertical error bar denote upper and lower height limits of data. Also included (at 12:30 and 17:30 UTC) is TKE from a tethered balloon-borne 3-D sonic operating at about 70 and $520 \mathrm{~m}$, respectively.

tion is generally much smaller (at least for the wind speeds encountered during the BLLAST field campaign).

\subsubsection{Height dependence of the dissipation term}

The dissipation rate of TKE is calculated in the model using the TKE length scale parametrization presented in Part 1:

$D=-\frac{E^{3 / 2}}{l_{\epsilon}}=-E^{3 / 2}\left(\frac{2.2}{z_{i}}+\frac{0.006}{z}\right)$.

The modeled profiles of dissipation for the afternoon of 20 June are shown in Fig. 6 as colored lines and shown with the near-surface dissipation as dots, UHF (ultrahighfrequency) wind profiler estimates from $175 \mathrm{~m}$ as lines with circles and estimates from aircraft as crosses at two height intervals of $75 \mathrm{~m}$ for 14:30 and 17:30 UTC. Near the surface, it is clear that the modeled dissipation decays more rapidly with height than it should because of too rapidly decaying in the shear production term, leaving too little shear-produced TKE $(=E)$ at $8 \mathrm{~m}$. The modeled dissipation in the boundary layer compares well to the aircraft estimates of dissipation and in order of magnitude to the UHF profiler estimates. However, the UHF profiler estimates shows a maximum at some height around 500-600 m, which the model and aircraft measurements do not show. It is not easily determined whether this often-seen feature in UHF profiler estimates is realistic. LES for this day did not show a pronounced maximum in dissipation rate (Darbieu et al., 2015b, a). The only way our simple parametrization could produce such a maximum in dissipation rate is if the TKE itself has a maximum at these heights. Vertical wind variance is well known to have a maximum at some height around $0.3-0.4 z_{i}$, whereas LES often produces a maximum of TKE closer to the surface (below $100 \mathrm{~m}$ ). Also, this simple model predicts such a feature on several of the more convective days at around $40-50 \mathrm{~m}$ but fails to do so on 20 June as we will see in a following subsection.

\subsubsection{Specification of initial neutral morning conditions}

The TKE budget equation is used to solve for the evolution of TKE from neutral morning conditions until the end of the afternoon. At the beginning of the simulation, for simplicity, we therefore assume the buoyant production term $B(z)$ to be zero at all heights. The shear production term $S(z)$ is calculated as before. The transport term is in this case only specified from the transport fraction and the shear term as $-S(z) T_{\mathrm{f}}$ to avoid an uncompensated positive layer in the upper part of the boundary layer.

Our treatment of initial conditions for dissipation $D(z)$ and turbulence kinetic energy $E(z)$ also differs from other time steps since there is no history of the flow to take into consideration at the first time step. Here, we first assume that the initial TKE tendency is negligible (such that $\frac{\partial E}{\partial t}=0$ ) and then solve for $D(z)$ based on the shear production and transport term; hence, $D(z)=-(S(z)+T(z))$. Then, we use this initial dissipation and estimate an initial TKE profile from $E(z)=\left(\frac{-z_{i} D(z)}{2}\right)^{2 / 3}$ such that the dissipation in the following time step will not obtain a large sudden jump when using the profile $E(z)$ to estimate dissipation using Eq. (16). It should be mentioned that height $z$ (or height above the displacement height in case of the forest) is ignored here for simplicity, but model tests showed small differences in results for the evolution of modeled TKE at midday and in the afternoon.

Our choices for initial conditions and modeling of morning transitions should be recognized only as a very crude attempt to represent a much more complex reality. Angevine et al. (2001), for instance, showed that during the morning transition a shallow mixed layer develops within the stable boundary layer and deepens rapidly when its potential temperature attains that of the residual layer. This indicates much more complexity for the growing phase of turbulence than what we assume here in our simple modeling. Initial conditions can, however, have limited influence on our results in midday and for afternoons if TKE remains close to its quasi-equilibrium value and also because there are many hours from our typical starting point around 05:00 UTC until midday.

\subsubsection{Height and time dependence of TKE}

The calculation of TKE tendency is essential for the time evolution of TKE. It was shown in Part 1 that TKE tendency is typically much smaller than the other budget terms, but if it were completely zero there could not be any evolution of the TKE so there is a difference between true steady-state and quasi-steady conditions.

In our model the evolution of TKE is determined by a finite-difference (forward in time) calculation with $1 \mathrm{~s}$ time step and $1 \mathrm{~m}$ vertical resolution from the other budget terms 
using the TKE budget equation.

$$
\begin{aligned}
& \frac{E\left(z, t_{n+1}\right)-E\left(z, t_{n}\right)}{\Delta t}= \\
& S\left(z, t_{n}\right)+B\left(z, t_{n}\right)+T\left(z, t_{n}\right)+D\left(z, t_{n}\right)
\end{aligned}
$$

The model can be considered semi-analytical in the sense that it contains only a numerical finite-difference timestepping scheme, but in all other ways is just a simple parametrization.

The resulting vertical profiles of TKE are shown in Fig. 7 for the afternoon of 20 June. In this figure, TKE at the small tower $(2.23-8.22 \mathrm{~m})$ was averaged and a standard deviation was calculated for each hour centered around 12:30, 14:30, 16:30 and 17:30 UTC. The result is shown with a vertical error bar indicating the minimum and maximum of the data. The same procedure was applied for the $60 \mathrm{~m}$ tower data, which consistently showed lower TKE levels compared to the near-surface TKE. The procedure was also applied to limited 3-D-sonic anemometer data from a sensor suspended from a tethered balloon (see Canut et al., 2016, for details). Data were available at $\approx 70$ and $520 \mathrm{~m}$ above ground at $12: 30$ and 17:30 UTC. At 14:30 and 16:30 UTC, the tower data have been slightly vertically displaced to better show the error bars without overlapping too much.

It is clear that the model produces TKE of the right order of magnitude and predicts the general reduction of TKE with height from the smaller tower to the $60 \mathrm{~m}$ tower. The decay of TKE in time may, however, be somewhat too rapid in comparison to measurements, as indicated by the low TKE levels at 16:30 and 17:30 UTC. However, the individual levels on the towers $(2.23$ and $61.4 \mathrm{~m})$ will be shown in time series plots in a later section which have quite reasonable levels of TKE for 20 June considering the large variability in $10 \mathrm{~min}$ values that occurs during these convective conditions.

The model only predicts an increase in TKE from the first model level to the second, due to the prescribed reduced shear production at the first grid point compared to the others. Otherwise, the model shows a decrease in TKE with height, which is not necessarily true at all height ranges. The measurements often show a small increase in TKE from 2.23 to $8.22 \mathrm{~m}$ (as is clear from Table B3 in Part 1), but consistently lower TKE levels at the $60 \mathrm{~m}$ tower imply a maximum of TKE somewhere close to the surface at a height on the order of tens of meters. As mentioned above, this simple model is capable of predicting a maximum of TKE near the surface at around 40-50 $\mathrm{m}$ for some of the more convective days of the field campaign. Then the model also often overestimates the TKE level at the $60 \mathrm{~m}$ tower. This could indicate that the maximum of TKE should be placed even lower than $40 \mathrm{~m}$.

\subsection{Differences and similarities compared to the model from Lenschow (1974)}

Figures 2-4 and 6 show the general shape of our modeled TKE budget terms which can be compared with another sim- ple TKE budget model from Lenschow (1974). To ease the comparison we have given a summary of the model from Lenschow (1974) in Appendix A and also plotted the resulting profiles for a convective case in Fig. A1 and a nearneutral case in Figure A2. In these figures we have also included vertical profiles from our model for 2 July from times when the same strength of overall stratification in terms of $z_{i} / L$ of -1000 and -1 occurred. It should be said that $z_{i}$ was 980 and $950 \mathrm{~m}$, respectively, for the 2 July cases and thus differs only $5 \%$ from the $1000 \mathrm{~m}$ used for the model from Lenschow (1974).

Concerning modeling of the buoyancy term in Lenschow (1974) the main difference is a further inclusion of some more fitting parameters for the shape of the vertical profile in the normalized height interval $0.87 \leq z / z_{i} \leq 1$ in their case (see Eq. A3 in Appendix A). Also, they make no prediction of TKE budget terms above the boundary layer depth, whereas we assume an entrainment zone that reaches above the height of minimum buoyancy flux.

For the shear production term Lenschow (1974) explored assuming a constant shearing stress (their model with this assumption is repeated in Appendix A) or a linearly decaying shear stress in the mixed layer. Lenschow (1974) also used a slightly different normalized wind gradient than us, but in general very similar results were obtained.

The profile of total transport shown in Figs. 4 and A1 compares qualitatively quite well to the modeled transport term in Lenschow (1974) with a negative layer in the lower boundary layer and a positive layer above. A linear profile shape in the mixed layer and a stronger curvature near the surface due to the effect of shear production is also present in both models (Fig. A1). The exact value of this term at specific heights differs, however, between these two models due to our inclusion of TKE budget terms also above the boundary layer depth $z_{i}$ and because of our specified near-surface transport fraction. In near-neutral conditions the model from Lenschow (1974) obtains negligible transport in comparison to shear production and dissipation terms (see Fig. A2b), whereas we retain more transport (most clearly seen near the surface) also in these conditions (with $T_{\mathrm{f}} \approx 0.36$ ). For very convective conditions we obtained $T_{\mathrm{f}}=0.46$, whereas Lenschow (1974) has a transport fraction $T_{\mathrm{f}} \approx 0.57$. This is of course an uncertain parameter that could be investigated more in future work. Data from Dupuis et al. (1997) may, for instance, suggest a value as large as 0.69 in convective conditions.

Qualitatively the vertical profile of dissipation is similar in our model compared to the model from Lenschow (1974) in convective conditions, illustrated in Fig. A1, with higher dissipation closer to the ground but with some important differences with increasing height. The dissipation approaches a constant value with height in the model from Lenschow (1974), whereas in our case it responds to the decreasing turbulence levels with increasing height. The exact level of dissipation in the two models close to the surface is also differ- 
ent under different stratification, mainly due to our different approaches to model vertical transport of TKE.

From Fig. A2 it is clear that the overall dissipation in our model can appear large in comparison to the model of Lenschow (1974) at the end of the afternoon. Our modeled profile is, however, taken from the very last minute before buoyancy flux becomes zero, when all surface forcings are quite small. The dissipation term in the mid-boundary layer is in fact only about $-2.6 \times 10^{-5} \mathrm{~m}^{2} \mathrm{~s}^{-3}$ at this point, but in a relative sense it is larger than in the model from Lenschow (1974). The reason for this larger dissipation term in our model in the overall boundary layer is because we linked our dissipation to the level of TKE. This introduces a slight memory effect of conditions that happened earlier in the simulation that is not present in the model from Lenschow (1974).

It is worth noting again that TKE tendency is assumed to be exactly zero in Lenschow (1974) and no prediction of TKE is therefore provided by this model. We included our TKE tendency term as blue lines in Figs. A1b and A2a and b. In convective conditions it is clear that the tendency term is very small compared to the other forcings. For our very close to neutral case at the end of the afternoon the TKE tendency is also small in actual units $\left(-2.5 \times 10^{-5} \mathrm{~m}^{2} \mathrm{~s}^{-3}\right.$ at $\left.0.5 z_{i}\right)$ but is not a negligible term in comparison to other budget terms in the mid-boundary layer. There it will be mostly determined by the available TKE that influences our modeled dissipation term.

\section{Observational data and processing}

\subsection{Description of data sets}

Our proposed model is based on a simplified TKE budget including idealized height-varying terms for shear production, buoyant production, transport and dissipation. It is driven with surface measurements (wind speed and fluxes) and boundary layer depth $z_{i}$. In this section, we describe the observational data set that is used (see Table 1) to drive the model and to evaluate it on TKE budget term estimates.

The BLLAST field campaign took place in June and July of 2011 in southern France at Plateau de Lannemezan, a plateau of about $200 \mathrm{~km}^{2}$ area, nearby the Pyrenees foothills, at equal distance from the Mediterranean Sea and from the Atlantic Ocean (about $200 \mathrm{~km}$ ). The surface is covered by heterogeneous vegetation: grasslands, meadows, crops, and forest. Several measurement sites were placed in the study area to obtain information of surface fluxes and winds from this heterogeneous landscape (Lothon et al., 2014).

Firstly, we will use wind speed and buoyancy flux from an $8 \mathrm{~m}$ tower (referred to as the "divergence site") and $z_{i}$ estimates from lidar measurements (all described in Part 1) to drive model simulations for nine IOP days. The lidar measurements were chosen due to slightly less fluctuating estimates compared to the UHF wind profiler estimates. On
26 June, $z_{i}$ from the UHF profiler was used because no lidar estimates were available. The hourly TKE budget results from the divergence site is also used to evaluate the model TKE budget terms and near-surface TKE. Furthermore, TKE from the $60 \mathrm{~m}$ tower was computed at three measurement heights $(29.3,45.8$ and $61.4 \mathrm{~m})$ with the same procedure as described in Part 1. Hence, 10 min TKE values were calculated before any further $1 \mathrm{~h}$ running mean procedure was applied. For evaluation purposes a limited set of data from a 3D sonic anemometer suspended from a tethered balloon was also used; see Lothon et al. (2014). For evaluation of boundary layer dissipation rate we also use estimates from a UHF wind profiler and measurements from full aircraft flight legs. These data sets are all found on the BLLAST database; see BLLAST (2015).

Secondly, as an exploration of the sensitivity in modeling results, we also use observed sensible and latent heat fluxes along with observed wind speed and temperature from five other land surface covers (moor, corn, grass, wheat and forest) to drive our TKE model. The fluxes are obtained from the uniformly processed data set by De Coster and Pietersen (2012) using the EC-PACK flux computation algorithm (Van Dijk et al., 2004). These flux time series are based on $30 \mathrm{~min}$ averaging periods and were also used by Hartogensis (2015) to derive area-averaged fluxes for the Plateau de Lannemezan area, based on the land use and complementary energy balance modeling for urban and bare-soil surfaces where no measurements were available. We will also show results for boundary layer dissipation rates based on such $2 \mathrm{~km}$ by $2 \mathrm{~km}$ area-averaged fluxes centered on the $60 \mathrm{~m}$ tower in Sect. 5. The specified data set including both observed time series, area-averaged fluxes, land use maps, documentation and quicklooks are found at the BLLAST website under the section "Area-averaged flux maps" in the BLLAST database.

In Table 1, we briefly summarize information about the surface data sets used. Here we also list the roughness length $z_{0}$ used in model simulations for the various sites. A value of $2 \mathrm{~cm}$ was estimated for the divergence site based on a period of reasonably steady winds in near neutral but slightly stable data (not morning or afternoon transition data). This value was also used for simplicity at all other sites and data sets except over the forest, where it was increased by a factor of 10 and a displacement height of two-thirds of an estimated average tree height of $20 \mathrm{~m}$ was used (Garratt, 1992).

We will show results from 9 of the 10 IOP days previously considered in Part 1. This is because there were no measurements available from the divergence site before 10:00 UTC on 19 June and we chose to consistently do simulations constrained by observations from the time of positive sensible heat flux in the morning until the end of the afternoon, defined from zero-buoyancy flux. This choice is to allow for the turbulence to build and decay during a long time period of sheared convective atmospheric conditions for each day. 
Table 1. Brief description of BLLAST measurement sites and data sets with the required buoyancy flux, winds and temperature for the modeling of TKE. Also listed are the roughness length $\left(z_{0}\right)$ and displacement height $(d)$ values in the model. For additional details see Lothon et al. (2014).

\begin{tabular}{lclccrr}
\hline Name & $\begin{array}{c}\text { Height } \\
\text { [ma.s.1.] }\end{array}$ & $\begin{array}{l}\text { Height } \\
{[\mathrm{ma.g.1.}]}\end{array}$ & $\begin{array}{c}\text { Sampling } \\
\text { rate [Hz] }\end{array}$ & $\begin{array}{r}\text { Aver. } \\
\text { period [min] }\end{array}$ & $\begin{array}{r}z_{0} \\
{[\mathrm{~m}]}\end{array}$ & $\begin{array}{r}\text { Disp. height } \\
d \text { [m] }\end{array}$ \\
\hline Divergence site & 591 & $(2.23,3.23,5.27,8.22)$ & 20 & 10 & 0.02 & 0 \\
60 m tower & 602 & $(29.3,45.8,61.4)$ & 10 & 10 & - & - \\
Grass site & 580 & 2.55 & 20 & 30 & 0.02 & 0 \\
Corn site & 645 & 2.93 & 20 & 30 & 0.02 & 0 \\
Moor site & 641 & 2.93 & 20 & 30 & 0.02 & 0 \\
Wheat site & 582 & 3.0 & 20 & 30 & 0.02 & 0 \\
Forest site (pine) & 620 & 31.55 & 10 & 30 & 0.2 & $\frac{2}{3} 20$ \\
$2 \mathrm{~km} \times 2 \mathrm{~km}$ & - & 8.22 (for wind speed) & - & 30 & 0.02 & 0 \\
\hline
\end{tabular}

\subsection{Description of data time series treatment}

The data sets described above all consist of estimates of different parameters, wind speed, buoyancy flux (or sensible and latent heat flux plus potential temperature that can be used to estimate buoyancy flux) and $z_{i}$ at different temporal resolutions. Before using these data to drive our TKE model we formed time series of $1 \mathrm{~s}$ temporal resolution in the following manner.

The time from positive sensible heat flux until zero buoyancy flux was estimated for each day and the time series manually checked. A few suspicious values in various time series were removed in this process. Then, a linear interpolation to $1 \mathrm{~s}$ values was applied followed by a $1 \mathrm{~h}$ running mean smoothing of the data. This procedure was adopted as we, especially for wind speed at these sites, found rapid variations in time and our intention is to attempt to model the more general slow decay of turbulence kinetic energy related to persistent changes in surface flux forcing and the slower trends observed in wind speed.

For boundary layer depth estimates, a $1 \mathrm{~h}$ running mean time series was formed in a similar way. Here, before linearly interpolating, a representative boundary layer depth value for the morning at the start of each simulation was subjectively estimated from the observed growing trend of $z_{i}$ later in the morning. This was done in spite of sometimes sparse observational estimates in the early morning. For the nine considered days starting at 20 June and ending at 5 July, the following nine initial values of $z_{i}$ were specified: $(150,250,200$, $150,200,150,100,200,200) \mathrm{m}$. This was needed to have a full time series of smoothly varying boundary layer depth evolution (i.e., $z_{i}(t)$ ) for the full time period of simulation, as required by the model.

\subsection{Treatment of dissipation rate from UHF wind profiler}

For evaluation of our model the data set of UHF wind profiler data described in Part 1 also includes estimates of TKE dissi- pation rate. It was available at an average temporal resolution of $5 \mathrm{~min}$ and a spatial resolution of $75 \mathrm{~m}$ starting at a height of $175 \mathrm{~m}$. We used the UHF profiler data from Site 1 (closest to the divergence site tower and $60 \mathrm{~m}$ tower; Lothon et al., 2014). These estimates of dissipation rate were based on Doppler spectral width following Jacoby-Koaly et al. (2002). Best estimates were formed from the median of the four oblique beams. We used the same software as described in Part 1 from Garcia (2010) to gap-fill and smooth the data set. The data were placed on a uniform time-height grid by observational minute and using the $75 \mathrm{~m}$ vertical resolution. Then, a smoothing parameter $S$ of $10^{-1}$ was used with five repeated iterations and an extra smoothing in time using a 15 min running mean value for each vertical level.

For evaluation, we also compared model estimates with UHF estimates and aircraft estimates of TKE dissipation rate from the Piper Aztec research airplane (Lothon et al., 2014). For that comparison, a further averaging of the UHF data for the same observational times as the corresponding flight legs followed by interpolation to the average height of the flight leg was performed.

To display the slower trends and evolution of TKE dissipation rate in a height time representation, the 5 or $15 \mathrm{~min}$ averaged data sets was considered still quite scattered and a running mean value of $1 \mathrm{~h}$ was applied for comparison with the modeled dissipation rate. This is reasonable here because we use a $1 \mathrm{~h}$ smoothed wind and surface flux time series as input to force the model and hence do not model the more temporary rapid variations in TKE budget terms.

\section{Evaluation of near-surface TKE and budget terms: nine IOP days}

In this section, we compare the simple model to measurements for nine IOP days studied in Part 1. The first objective is to investigate the simple model's ability to predict a reasonable near-surface TKE and TKE budget evolution for the diverse set of conditions that occurred on these 9 days despite 

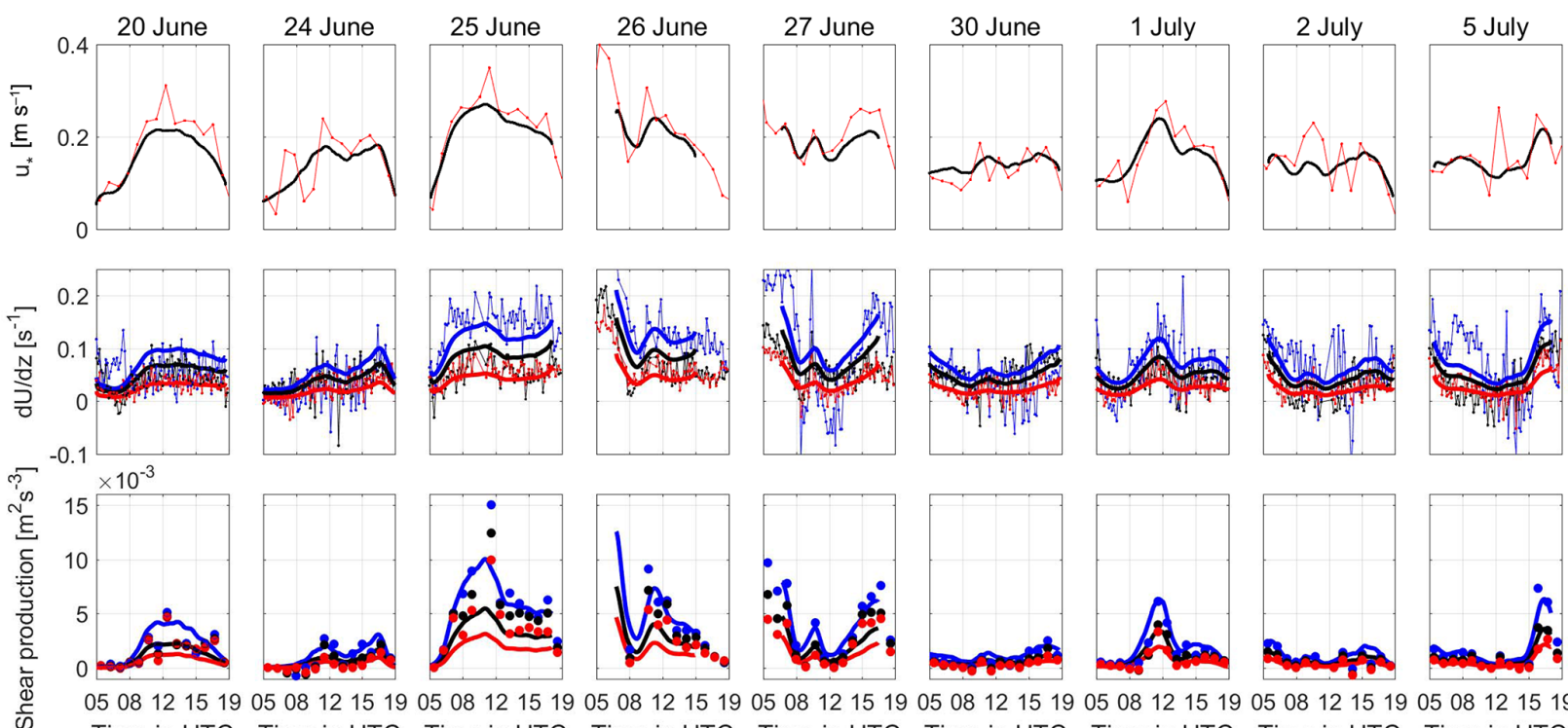

Time in UTC Time in UTC Time in UTC Time in UTC Time in UTC Time in UTC Time in UTC Time in UTC Time in UTC

Figure 8. The upper row shows the modeled stability-corrected friction velocity (black line) and observations at $3.23 \mathrm{~m}$ (red line with dots) for nine simulated days. The simulations are performed only for unstable conditions, whereas measurements are shown between 05:00 and 19:00 UTC on all days. The middle row shows the measured wind gradient at 2.73, 4.23 and $6.75 \mathrm{~m}$ as thin blue, black and red lines with dots, and the corresponding modeled wind gradients is shown as full blue, black and red lines for the 3, 4 and 7 m model levels. The lower row shows the modeled shear production interpolated to the measurement heights of the three upper levels on the small tower as full lines. The corresponding hourly budget shear production values are shown as blue, black and red circles when available under the conditions discussed in Part 1.

its deficiencies. The second aim is to discuss why the model produces unreasonable results. This indicates potential focus areas for future model improvement.

\subsection{Evaluation of near-surface TKE budget terms}

The upper row of Fig. 8 shows the model's stability-corrected friction velocity $u_{*}$ as black lines and observations at $3.23 \mathrm{~m}$ as red lines with dots. It is clear that our approach gives reasonable estimates of $u_{*}$ on many occasions, but it also misses some low and especially high values that occur for periods of 1 or $2 \mathrm{~h}$. Further, the modeled friction velocity, based on mainly the mean wind speed, does not always reflect this observed variability and produces a smoother evolution of $u_{*}$ for each day.

The middle row of Fig. 8 shows the measured wind speed gradient based on $10 \mathrm{~min}$ values as thin colored lines with dots and the modeled wind speed gradient as thicker colored lines. In this case, it is clear that wind gradients shift rapidly and the model, as a consequence of our simplifications, captures only some of the low-frequency variability in the observations. This is, however, not always the case (see, e.g., 27 June as well as 2 and 5 July). The too rapidly decaying shear production term with increasing height in comparison to measurements stems from both deviations in the assumed wind gradient and height dependence of frictional stress.

The observed hourly shear production is shown in the lower row of Fig. 8 with colored dots. The model (thick lines) does capture some of the day to day variability, but the smooth model results do not capture all of the individual hourly variability seen in the measurements. Furthermore, shear production tends to be underestimated at times, with higher shear production such as on 25 and 26 June. This underestimation is more severe at $8 \mathrm{~m}$ as a consequence of the shear production height dependence, which decays too rapidly with height. This is seen on 25 and 26 June, at the end of the afternoon of 27 June, and to some extent on 20 June and in the middle of the day on 1 July. Underestimation of the generation of TKE from missing periods of high wind speed is natural because the source of TKE depends on $u_{*}^{3}$. The importance of these excursions will, however, also depend on how quickly departures from quasi-equilibrium are damped.

The observed and modeled near-surface buoyant production is shown in the upper row of Fig. 9 and is in general a good (albeit smoothed) representation of the measurements. On 20 June at 12:30 UTC, the model underestimates the measured buoyancy production at 5.27 and $8.22 \mathrm{~m}$, as has already been noted in Fig. 3. On 30 June, which had variable cloud cover, similar errors are also seen, but otherwise in most cases the differences between model and measurements are smaller for this more directly forced budget term.

The middle row of Fig. 9 shows the modeled and observed transport, which show significantly larger scatter in observed values compared to the buoyant production and larger indi- 


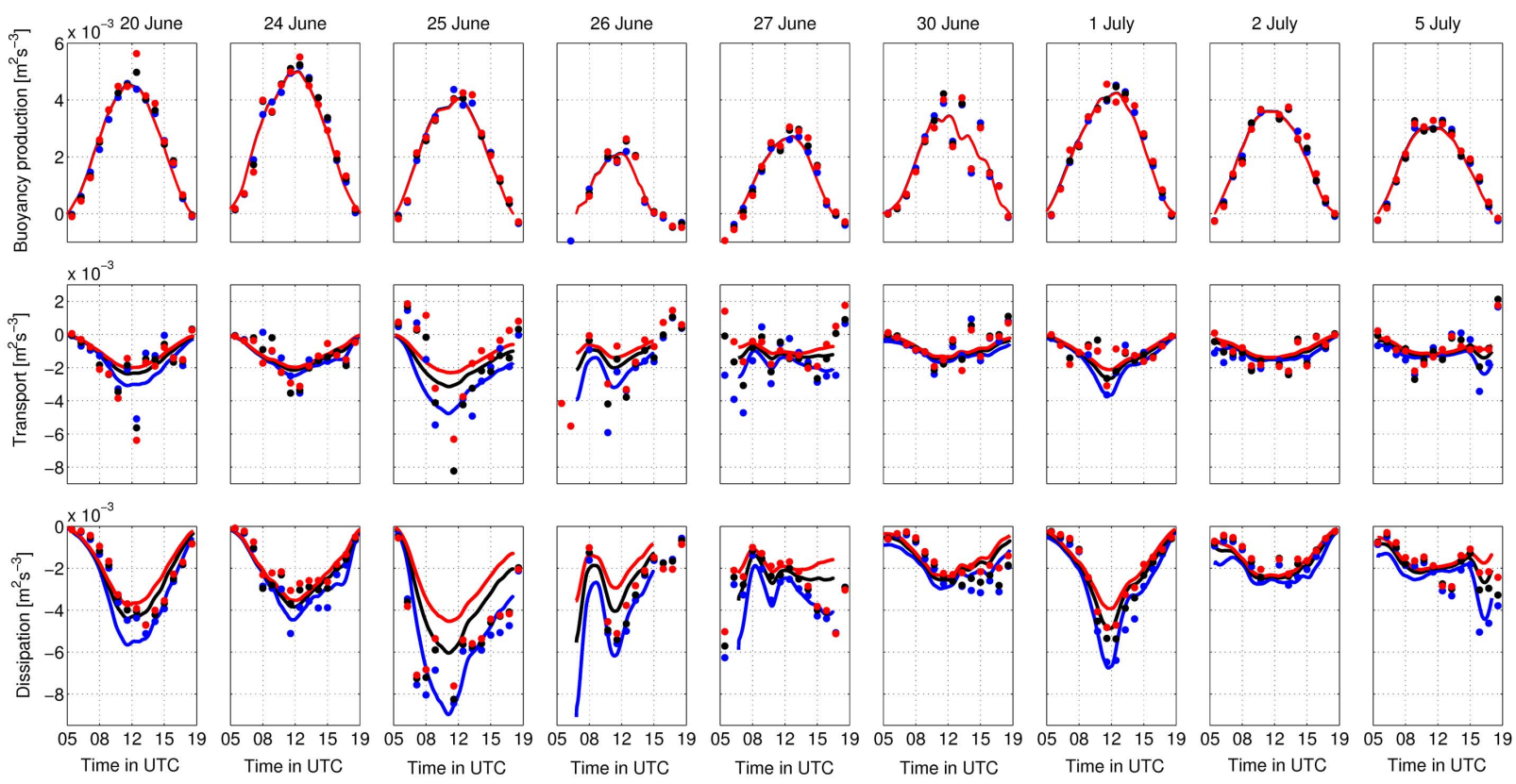

Figure 9. The upper row shows the observed hourly averaged buoyancy production at 2.23, 5.27 and $8.22 \mathrm{~m}$ (filled blue, black and red circles) and the corresponding model buoyancy production (lines). The middle row shows the corresponding observed and modeled values for transport. The lower row shows the observed and modeled values of dissipation.

vidual discrepancies between model and measurements. Particularly on days with more wind, the scatter is larger, such as on 20 and 25-27 June. As discussed, it is challenging for the model to capture the shear production well on an hourly basis, and the modeled transport also has a smoother evolution in time than the observations.

The lower row shows the observed and modeled dissipation. The model captures much of the day to day as well as hourly variability, but at times of strong shear production, it underestimates at the $8.22 \mathrm{~m}$ level. This is observed on 25 , 26 and the afternoon of 27 June as well as in the middle of the day on 1 July. The model also overestimates dissipation somewhat on 2 July and during the morning period of 5 July until around 12:00 UTC.

All these observed errors in the modeled TKE budget terms, which may at times be considered quite small, can lead to problems in the prediction of the TKE as any systematic errors can cause an accumulated effect for the TKE prediction.

\subsection{Evaluation of near-surface TKE}

We find the modeled results of TKE at the $2.23 \mathrm{~m}$ level and $61.4 \mathrm{~m}$ level presented in Fig. 10 quite encouraging and indicative that our reported budget term expressions can reproduce the overall level of observed TKE well. There are, however, obvious discrepancies between the model and measurements that need to be discussed further.
For the $2.23 \mathrm{~m}$ level, shown in the lower row of Fig. 10, the model underestimates the TKE on 8 out of 10 days at the beginning of the simulation up until around 08:00 or 09:00 UTC (at least). This is probably mostly related to uncertainty in the way we define initial profiles of TKE for neutral morning conditions. The level of TKE at $2 \mathrm{~m}$ during midday is relatively well captured on many of the days but too low on 2 and 5 July. On 2 July and the morning of 5 July this could be due to a slight overestimation of near-surface dissipation. On 5 July there are also a few hours of an observed positive transport term at some heights (and small at other heights), implying a potential import of near-surface TKE, which, if it did occur, cannot be captured by the simple model. This was also observed very temporarily on 27 and 30 June, which, as discussed in Part 1, could be related to variable cloud cover and/or uncertainty in dissipation estimates. With this one-dimensional model, it is difficult to draw conclusions regarding the import of TKE from above or by horizontal advection. On the morning of 25 June, however, there are several hours with observed positive values of the transport term at all measurement heights, and this may have additionally contributed to an underestimation of nearsurface TKE in the morning of this day.

At $61.4 \mathrm{~m}$, the TKE level is underestimated on 25 and 26 June and at the end of the afternoon on 27 June. It is likely a consequence of too rapidly decaying shear production with height. The model also tends to overestimate TKE on some days with higher buoyancy production (e.g., 24 and 30 June, 1 and 2 July). It is unclear, however, to what extent the ob- 

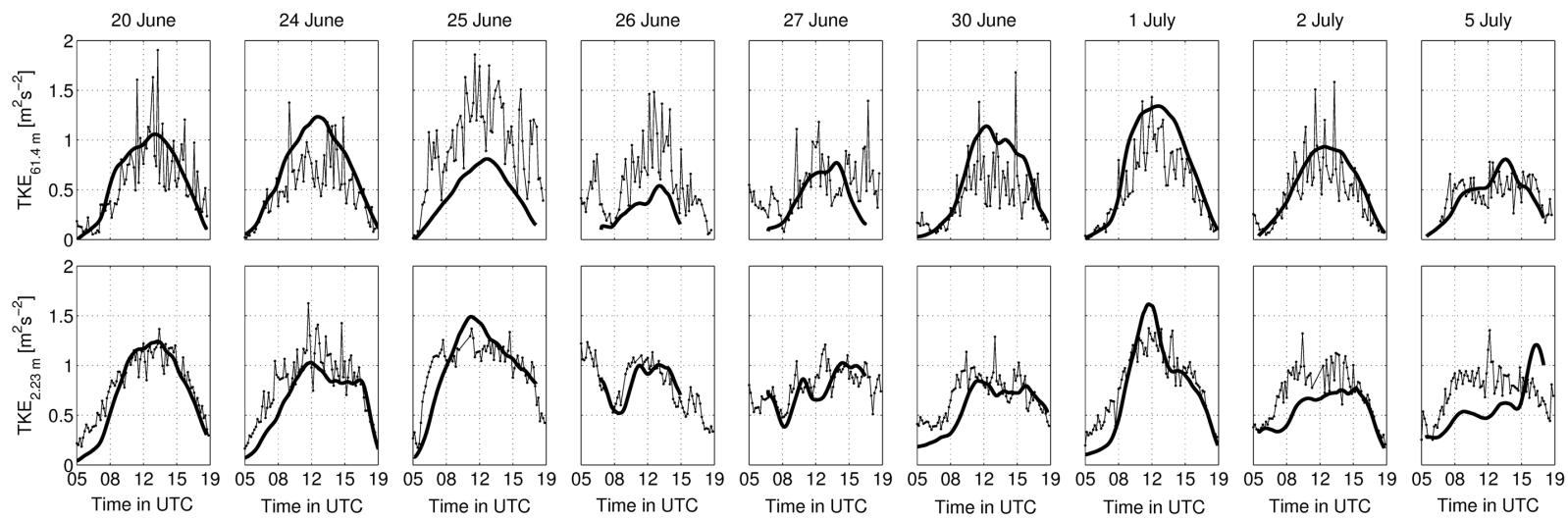

Figure 10. The lower row shows the modeled TKE interpolated to $2.23 \mathrm{~m}$ (full lines) and observations (thin lines with dots). The upper row shows TKE at the $61.4 \mathrm{~m}$ level.

served differences at $60 \mathrm{~m}$ should be related to issues with the model or related to differences in fluxes and wind that occur at different surfaces in the landscape surrounding the $60 \mathrm{~m}$ tower. It may of course be that the observed flux and wind at the divergence site is not always representative of a height of $60 \mathrm{~m}$. A flux footprint analysis (Hartogensis, 2015) for the $60 \mathrm{~m}$ tower indicates that grass and moor (with relatively low fluxes similar to those observed at the divergence site) dominate the fluxes in unstable conditions at the $60 \mathrm{~m}$ tower, usually accounting for about 65 to $85 \%$ of a reconstructed flux. With the remaining reconstructed flux related mainly to forest, urban and bare soil land surfaces. When the wind comes from the north or northeast the flux is especially dominated by grass and moor conditions (Hartogensis, 2015), but this changes when the wind comes from the east. Interestingly we observe that the model underestimates the TKE especially when the wind in the lower convective boundary layer and near the surface is from the east, rather than typically from the north or northeast for the rest of the time. The easterly flows happen on 25 and 26 June, in the late afternoon of 27 June and in the morning of 5 July (see wind direction close to the surface in Part 1, Fig. 3). All these periods correspond to an underestimated TKE in the model at $61.4 \mathrm{~m}$. This could be linked with the presence of a band of forest to the east and the Lannemezan village behind, and that either the flux or the shear production that we use does not represent their effect. It could also be due to advected TKE from the east. These effects related to heterogeneity in the landscape in combination with shifting wind direction also cause the reconstructed flux (at the $60 \mathrm{~m}$ tower) to have a variable contribution from different surface land covers on both a daily and hourly basis. A flux footprint analysis may, however, not be directly translated to apply for a variable such as TKE. Therefore, we mainly conclude that the model performs reasonably well at $2.23 \mathrm{~m}$ and less well, but still with the right order of magnitude, for TKE at $61.4 \mathrm{~m}$.

\section{Sensitivity test of surface boundary conditions: influence on boundary layer dissipation rate and the formation of a pre-residual layer}

As an exploration into the sensitivity of model results to different observed fluxes and winds over different surface types, in Fig. 11 we show modeled dissipation rate for 30 June from five simulations over corn, moor, forest, wheat and grass with available measurements. Also shown are model results for the divergence site and using a $2 \mathrm{~km}$ by $2 \mathrm{~km}$ area-averaged flux, as well as the observed dissipation rate from a UHF wind profiler. On this day, there was no distinguishable bias between dissipation rates from the UHF profiler and aircraft measurements (not shown here) and therefore a comparison of the overall modeled boundary layer dissipation with the observations from the UHF profiler is reasonable to make.

The divergence site tower measurements show very similar low fluxes as observed over grass and moor, and for this day also corn. This is in contrast to the higher observed fluxes over forest and wheat. The surface flux over the grass, moor, corn and divergence sites yielded the most similar levels of dissipation rate compared to the observations on this day, whereas other surface types lead to higher levels of dissipation rate. Based on energy balance modeling, fluxes of urban and bare soil land covers were also determined to be high, corresponding roughly to the forest level (Hartogensis, 2015). In particular, we believe that the urban land cover used likely overestimate the real flux from the villages considered here, which have much vegetation between the houses. Therefore, using area-averaged fluxes over a $2 \mathrm{~km} \times 2 \mathrm{~km}$ or $10 \mathrm{~km} \times 10 \mathrm{~km}$ area of the surroundings leads to higher estimates of boundary layer TKE and dissipation rates compared to using the divergence site observations.

We note that the model may overestimate boundary layer dissipation somewhat for 30 June and turbulence may not be as capped in value in the model as indicated from the UHF profiler. The simple model presented here lacks ele- 

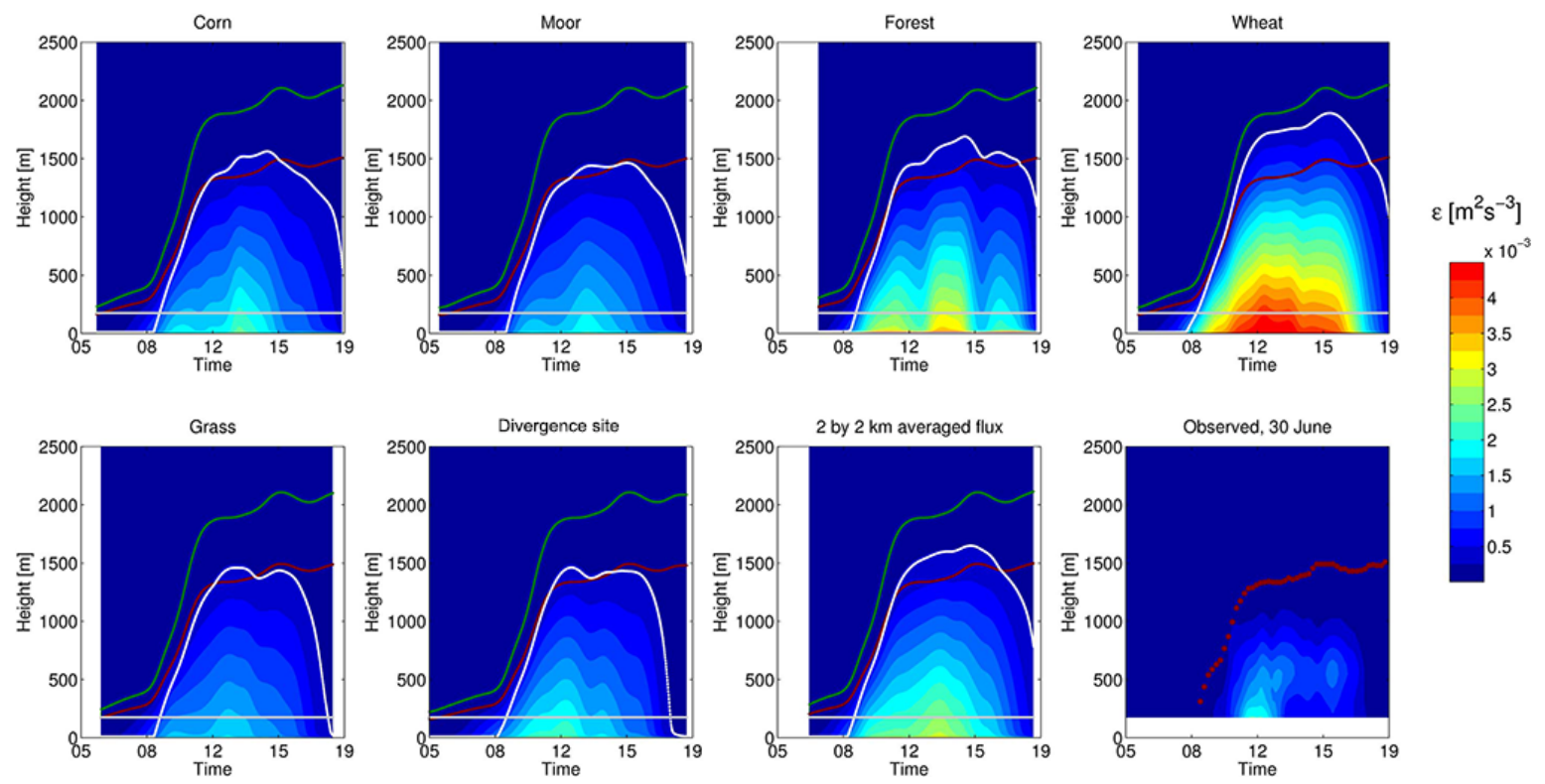

Figure 11. Modeled boundary layer dissipation rate for 30 June for six simulations over different surfaces with available near-surface measurements during the BLLAST field campaign, as well as a simulation driven by a $2 \mathrm{~km} \times 2 \mathrm{~km}$ averaged flux. The lower right panel shows the observed dissipation rate from the UHF wind profiler between 175 and $2000 \mathrm{~m}$. The red line or dots indicate model and observed boundary layer depth, the green line indicates the model height of no turbulence, and an isoline for TKE equal to $0.3 \mathrm{~m}^{2} \mathrm{~s}^{-2}$ is included as a white line. A horizontal gray line is also included at $175 \mathrm{~m}$ to show the lowest level available from the UHF wind profiler.

vated wind shear in the entrainment zone, which may lead to an underestimation of dissipation rate and TKE in the upper parts of the boundary layer. Elevated shear may, however, also affect the entrainment process and the entrainment parameter which here has been simply taken as a constant value of -0.15 based on a study for 20 June. Model tests changing this value to -0.3 showed reduced levels of TKE and dissipation rate in the upper parts of the boundary layer, but with otherwise similar results and only a small impact in the lower part of the boundary layer.

An apparently important result from this study is that the modeled decay of dissipation rate and TKE occurs first at the upper part of the boundary layer during the afternoon as a response to the diminishing surface buoyancy flux forcing. This may, of course, in reality be prevented by the presence of elevated wind shear, but on most of the days it is also observed by the UHF profiler. Grimsdell and Angevine (2002) and Lothon et al. (2014) also revealed, with remote sensing observations, a decay of TKE dissipation rates from top to bottom (although not systematically). This was also used as a way to define a top of the turbulent boundary layer in the afternoon transition in numerical weather prediction models (Couvreux et al., 2016). In Fig. 11 the white line shown is an isoline for TKE (corresponding to $0.3 \mathrm{~m}^{2} \mathrm{~s}^{-2}$ ), which is of course an arbitrarily chosen value, but it indicates low turbulence levels. It is instructive from a conceptual point of view to consider these conditions with low turbulence levels during the afternoon transition as a pre-residual layer. It forms when the boundary layer turbulence adjusts to the weaker buoyancy flux forcing from the surface. It is capped by the mixed-layer inversion and does not reach the surface, except possibly very near neutral stratification at the end of the afternoon or the beginning of the evening transition. It is useful to introduce this concept of a pre-residual layer as we consider that it is an important part of explaining the onset turbulence conditions for the nocturnal residual layer.

The residual layer is defined as the statically neutral layer, characterized by weak sporadic turbulence, that lies above the stable boundary layer and below the capping inversion, separating the boundary layer flow from the free atmosphere. By definition, it begins to develop only after the surface begins to stably stratify. Therefore, it is useful to also name the region of weak turbulence that exists during unstable conditions preceding the residual layer as the pre-residual layer. It is within these continuously weakening afternoon turbulence conditions that many things characterizing the turbulence are changing, such as the shape of spectra of vertical wind velocity and integral length scales (Darbieu et al., 2015b). Darbieu et al. (2015b) showed with LES and measurements that change occurs first in the upper part of the boundary layer during the later stages of the afternoon transition, and the higher within the ABL, the stronger the spectra changes. These observations may hence be considered to have taken place in the pre-residual layer and may potentially provide other ways to define and characterize it more exhaustively in the future. 


\section{Parameter exploration for near-surface TKE}

In this section, we first discuss the setup and results of modeled near-surface TKE for the afternoon based upon complementary idealized numerical simulations in Sects. 6.1-6.5. Secondly, we comment upon our results in relationship to turbulence decay laws in Sect. 6.6. We also compare our numerical model results to a simplified analytical expression assuming quasi-stationary turbulence in Sect. 6.7. Here, we also illustrate and discuss the added value of our modeling efforts taking into account variations in wind or $u_{*}$ compared to only taking into account of $w_{*}$ as a scaling variable for TKE.

\subsection{Setup of different scenarios}

The sensible heat flux used in these model runs are provided by a cosine function as in Sorbjan (1997) and several other earlier and subsequent studies:

$H_{\mathrm{cos}}\left(t^{\prime}\right)=H_{\max } \cos \left(\frac{2 t^{\prime}}{\pi \tau_{\cos }}\right)$.

Here, $H_{\max }$ defines the maximum sensible heat flux at midday, and the decay timescale $\tau_{\cos }$ defines the length of the period with positive sensible heat flux (half of which covers the afternoon period). For simplicity, we chose a zero latent heat flux in these idealized simulations.

For boundary layer depth, we specify a very simple sine function increase of $z_{i}$ from a neutral morning value $z_{i \min }$ to a midday value $z_{i_{\max }}$ by

$z_{i}\left(t^{\prime}\right)=z_{i_{\min }}+\left(z_{i_{\max }}-z_{i_{\min }}\right) \sin \left(\frac{2 t^{\prime}}{\pi \tau_{\cos }}\right)$,

which is then kept constant for the afternoon. This prescribed evolution is of course a simplification, as discussed in Sect. 2.3.5 the morning transition can have much complexity as shown in Angevine et al. (2001). Our results for midday and afternoon was, however, relatively insensitive to this modeling choice.

The complementary idealized numerical simulations have been performed by systematically varying a studied parameter while keeping all the other variables specified according to a reference simulation. We begin by providing the details of the reference simulation. For this simulation, we keep the wind speed constant at $2 \mathrm{~ms}^{-1}$ throughout the whole simulation. However, this does not mean a constant $u_{*}$ value in the case of unstable stratification because of the stability correction of $u_{*}$ and flux gradient relationship described in Sect. 3.2. A mean temperature of $20^{\circ} \mathrm{C}$ with a corresponding density of air $\rho=1.205 \mathrm{~kg} \mathrm{~m}^{-3}$ and specific heat capacity is used, as well as a roughness length $z_{0}=0.02 \mathrm{~m}$ and entrainment parameter $B_{\mathrm{E}}=-0.15$. The morning boundary layer depth $z_{i_{\min }}$ was kept at $150 \mathrm{~m}$ in all simulations. The values of the specific parameter settings which we vary for the reference simulation are given in column 3 of Table 2 .
We conduct six different types of model experiment test runs denoted by AL (afternoon length), BLD (boundary layer depth), SH (sensible heat flux), $U_{\mathrm{c}}$ (constant mean wind speed), $U_{\text {inc }}$ (increasing wind speed), and $U_{\text {dec }}$ (decreasing wind speed) runs. For each of these, one variable of interest is changed. For the AL, BLD and $\mathrm{SH}$, it is simply the variables $\tau_{\cos }, z_{i_{\max }}$ and $H_{\max }$ that are systematically varied. For the $U_{\text {mean }}$ runs, it is a specified constant wind speed throughout the entire simulation that is varied. For the $U_{\text {inc }}$ runs, we instead keep the wind speed at zero until midday and then increase it linearly to a specified value $U_{1}$ at the end of the afternoon (which is a normalized time of 1 after dividing by the afternoon length). In this way we study one of the simplest cases of a time-varying wind speed for the afternoon. Similarly, in the $U_{\text {dec }}$ runs we instead let the wind speed be constant for the morning period until midday at a value denoted $U_{0}$ and then specify a linear decrease in wind speed to a value of zero at the end of the afternoon. In columns 4 and 5 of Table 2 and continued in Table 3, we list the parameter settings for our model experiment test runs. For shortening of the table, we denote our $U_{1}$ and $U_{0}$ settings with $U_{\mathrm{c}} *$, which means the same numerical values from 0 to $3 \mathrm{~m} \mathrm{~s}^{-1}$ were used as in our constant mean wind speed runs $U_{\mathrm{c}}$. It should be noted that these settings represent a range of conditions encountered during the BLLAST field experiment.

\subsection{Results from varying the afternoon length}

Only $2 \mathrm{~m}$ results from our idealized modeling will be discussed since the model compares well with measurements of TKE at $2.23 \mathrm{~m}$ in the previous section and less well at other heights. Our AL runs indicated that varying the afternoon length played a small role on the near-surface TKE results (not shown here). After normalization with the afternoon length, the results collapsed to within $1-2 \%$ of each other with a slight tendency that longer afternoon lengths resulted in lower TKE. The differences are small enough that they may be a result of the simple explicit time-stepping routine or other modeling aspects (e.g., rounding the prescribed smoothed boundary layer depth $z_{i}$ to the nearest grid level value). This is not contradicting the result of van Driel and Jonker (2011) considering that the afternoons studied here are $2 \mathrm{~h}$ or longer and hence long in comparison to the largeeddy turnover time of turbulence.

\subsection{Results from varying the boundary layer depth}

Our BLD runs showed an increase in midday TKE from about 0.8 to $1.3 \mathrm{~m}^{2} \mathrm{~s}^{-2}$ for a change in prescribed boundary layer depth from 400 to $1600 \mathrm{~m}$ and smaller differences were observed at the end of the afternoon. Normalization of the modeled TKE with the midday TKE value $\left(\mathrm{TKE}_{0}\right)$ collapsed the data very well (not shown here, but to within $1 \%$ ) and therefore differences due to boundary layer depth (when 
Table 2. Simulation settings for model experiment test runs.

\begin{tabular}{lllll}
\hline Name & Parameter & Reference run & AL runs & BLD runs \\
\hline Afternoon length & $\tau_{\cos }[\mathrm{h}]$ & 6 & $(2,4,6,8,10)$ & 6 \\
Maximum sensible heat flux & $H_{\max }\left[\mathrm{W} \mathrm{m}^{-2}\right]$ & 200 & 200 & 200 \\
Wind speed at $10 \mathrm{~m}$ & $U_{10}\left[\mathrm{~m} \mathrm{~s}^{-1}\right]$ & $U_{\text {mean }}=2.0$ & $U_{\text {mean }}=2.0$ & $U_{\text {mean }}=2.0$ \\
Afternoon boundary layer depth & $z_{i_{\max }}[\mathrm{km}]$ & 1.0 & 1.0 & $(0.4,0.6,0.8,1.0,1.2,1.4,1.6)$ \\
\hline
\end{tabular}

Table 3. Simulation settings for model experiment test runs.

\begin{tabular}{lllll}
\hline Parameter & SH runs & $U_{\mathrm{c}}$ runs & $U_{\text {inc }}$ runs & $U_{\text {dec }}$ runs \\
\hline$\tau_{\text {cos }}[\mathrm{h}]$ & 6 & 6 & 6 & 6 \\
$H_{\max }\left[\mathrm{W} \mathrm{m}^{-2}\right]$ & $(0,50,100,150,200,300,400,500)$ & 200 & 200 & 200 \\
$U_{10}\left[\mathrm{~m} \mathrm{~s}^{-1}\right]$ & $U_{\text {mean }}=2.0$ & $U_{\mathrm{c}} *=(0,0.5,1,1.5,2.0,2.5,3.0)$ & $U_{1}=U_{\mathrm{c}} *$ & $U_{0}=U_{\mathrm{c}} *$ \\
$z_{i_{\max }}[\mathrm{km}]$ & 1.0 & 1.0 & 1.0 & 1.0 \\
\hline
\end{tabular}

about constant during the afternoon) are small in such a representation.

\subsection{Results from varying the sensible heat flux}

In the lower row of Fig. 12, we show the TKE from the test runs listed in Table 3 and in the upper row the results after normalization with afternoon length and midday TKE. From left to right the $\mathrm{SH}, U_{\mathrm{c}}, U_{\text {inc }}$ and $U_{\mathrm{dec}}$ runs are shown. Starting with the SH runs, testing the variation in TKE due to changed sensible heat flux forcing, it is clear (from the lower left plot) that higher TKE levels are found at midday as a more convective boundary layer is modeled. From the upper left panel, it is also clear that the turbulence maintained by wind shear at the end of the afternoon becomes, as a percentage, a smaller amount of the midday TKE value for increasingly convective conditions. Due to slight memory effects, the actual TKE level at the end of the afternoon can, however, be slightly higher in the convective simulations than in the completely neutral case with constant wind speed (full black line). The neutral case have a steady-state TKE level due to a balance of shear production, transport and dissipation maintained throughout the simulations. This memory effect caused by convectively generated turbulence not being completely dissipated at the end of the afternoon is, however, small. In this case and this close to the surface it is less than $15 \%$ of the TKE in the neutral simulation.

\subsection{Results from varying the wind speed}

Turning now to the cases of $U_{\mathrm{c}}$ simulations where the wind speed is systematically increased from 0 to $3 \mathrm{~m} \mathrm{~s}^{-1}$ (second plot to the left in the lower row of Fig. 12), we find that TKE levels increase with increasing wind speed, as expected. From the corresponding upper panel, it is also clear that percentage decay of TKE during the afternoon decreases with increasing wind speed. In the limit of very high and con- stant wind speed, we would (using this normalization) approach the neutral steady-state solution where buoyancy flux no longer matters (corresponding to the zero buoyancy flux case of the upper left panel). Hence, it is the relative amount of shear and buoyancy that determines this decay percentage in the case of constant wind speed.

Results from the slightly more complicated situation of TKE evolution in the case of a linearly increasing wind speed during the afternoon from zero to a specified value at the end of the afternoon ( $U_{\text {inc }}$ runs) are shown in the lower second plot from the right in Fig. 12. The midday TKE level is the same as for the zero wind speed simulation, and successively increasing the wind speed decreases the turbulence decay for the afternoon. For the two windiest cases, an increase in TKE during the afternoon is observed instead of a decay. At the very end of the simulations, a slight drop in TKE can be seen even for these cases. This is due to a decrease in the stabilitycorrected $u_{*}$ value as we approach neutral conditions, but this effect is small compared to the general increase in TKE due to the increasing shear production. Normalization of these cases using a midday TKE value is shown in the upper panel. The normalization does not collapse the data simply because the midday TKE level was the same, and it was the changes that occurred during the afternoon that caused the difference in TKE levels at the end of the afternoon.

Finally, in the plots on the right we show the results from our $U_{\text {dec }}$ runs in which we decrease wind speed linearly to zero from some value (which was held constant up until midday). A decrease in wind speed is often the typical situation for variation in surface layer wind speed during the afternoon (Wingo and Knupp, 2015). In this case the midday value for TKE is the same as in our $U_{\mathrm{c}}$ runs, where wind speed was held constant throughout the entire simulation. The difference is that shear production decreases continuously during the afternoon and hence at the end of the afternoon there is no wind to maintain turbulence and significantly lower turbu- 

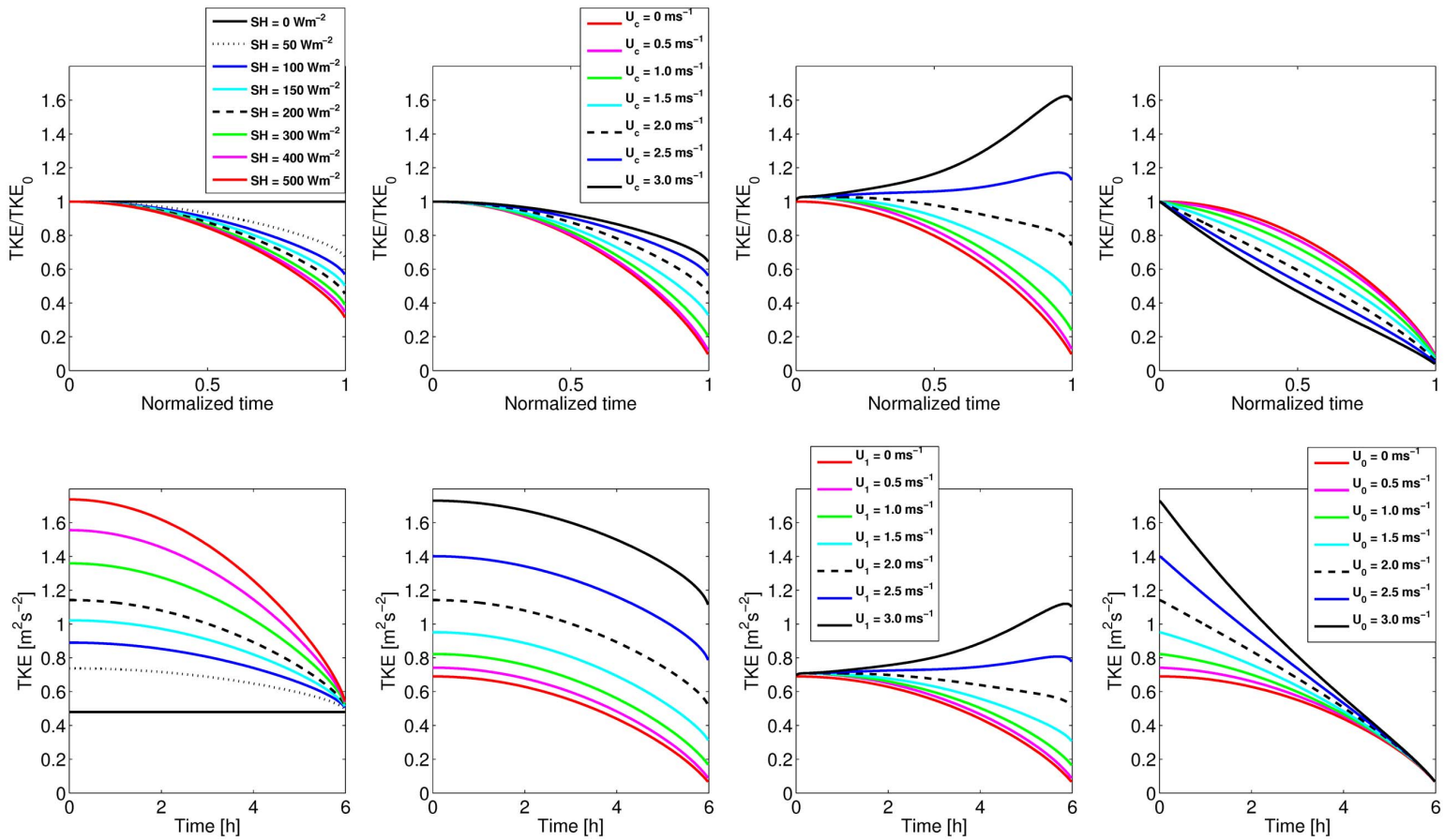

Figure 12. The lower row shows the evolution of modeled TKE at $2 \mathrm{~m}$ for the afternoon period in four sets of numerical simulations. In the upper row, the same simulation results are shown after dividing the TKE by the midday TKE value for each simulation and normalizing time with the length of the afternoon (here $6 \mathrm{~h}$ ). From left to right the simulation results shown are from the $\mathrm{SH}, U_{\mathrm{c}}, U_{\mathrm{inc}}$ and $U_{\mathrm{dec}}$ numerical experiments and the legends show the relevant variable value set for each simulation. The legends shown apply to both upper and lower panels.

lence levels are found at the end of the afternoon. In this case, a normalization with the midday TKE value will of course cause a gathering of the curves, but the most windy midday situation (black full line), which has the largest change in production of TKE from midday until the end of afternoon, clearly has the fastest percentage decay of TKE. This situation was faster than the approximately linear decay seen for the simulation with $U_{0}=2 \mathrm{~m} \mathrm{~s}^{-1}$ marked with a dashed black line.

\subsection{Comment upon turbulence decay laws}

The result of an approximately linear decay of TKE in time can be very instructive to consider in relationship to previous modeling results from Nadeau et al. (2011), Sorbjan (1997) and Nieuwstadt and Brost (1986). They chose to describe their results with a logarithmic representation for both TKE and time and discuss the turbulence decay in terms of a decay exponent parameter $\alpha$ such that a time dependence of normalized TKE is related to $t^{\alpha}$. Whereas early LES studies (Nieuwstadt and Brost, 1986; Sorbjan, 1997) lead to decay exponents of -1.2 and -2 , surface layer measurements (Nadeau et al., 2011) and recent LES (Rizza et al., 2013) pointed out the existence of a range of exponents (e.g., -2 through at least -6$)$. In the case of a linear change in TKE with time such that $\mathrm{TKE}=k t+\mathrm{TKE}_{0}$, we obtain $y=\ln (\mathrm{TKE})=\ln \left(k e^{x}+\mathrm{TKE}_{0}\right)$ with $x=\ln (t)$ and the decay parameter then becomes $\alpha=\frac{\partial y}{\partial x}=1-\frac{\mathrm{TKE}_{0}}{\mathrm{TKE}_{0}+k t}$. This shows that $\alpha$ becomes a function of time for the simple case of a linear change in TKE with time. Furthermore, two values of observed $\alpha$ during a single afternoon such as -2 and -6 can occur without necessarily implying a different decay rate of TKE in terms of $\mathrm{m}^{2} \mathrm{~s}^{-3}$ at those times. Therefore, and in the light of the above simulation results, which show both faster and slower than linear decay rates (and even increasing TKE for afternoons with increasing wind speed), we conclude that, at heights near the surface, there is unlikely any general simple decay exponent value $\alpha$ for turbulence kinetic energy.

All results presented here concern the TKE decay near the surface during the afternoon transition with still unstable conditions. Nadeau et al. (2011) pointed out the necessity for LES to confirm whether their observed surface layer results persist after averaging over the boundary layer depth. This was done in Rizza et al. (2013) and, in addition, Darbieu et al. (2015b) studied the height variation in TKE using LES and measurements. Our conclusions about the limitations of simple decay exponent values for near-surface TKE could similarly be tested with LES. Pino et al. (2006) showed for bulk-averaged TKE that shear generation can give reduced TKE decay, and we showed with near-surface measurements (Part 1) that with significant shear the TKE in the afternoon 

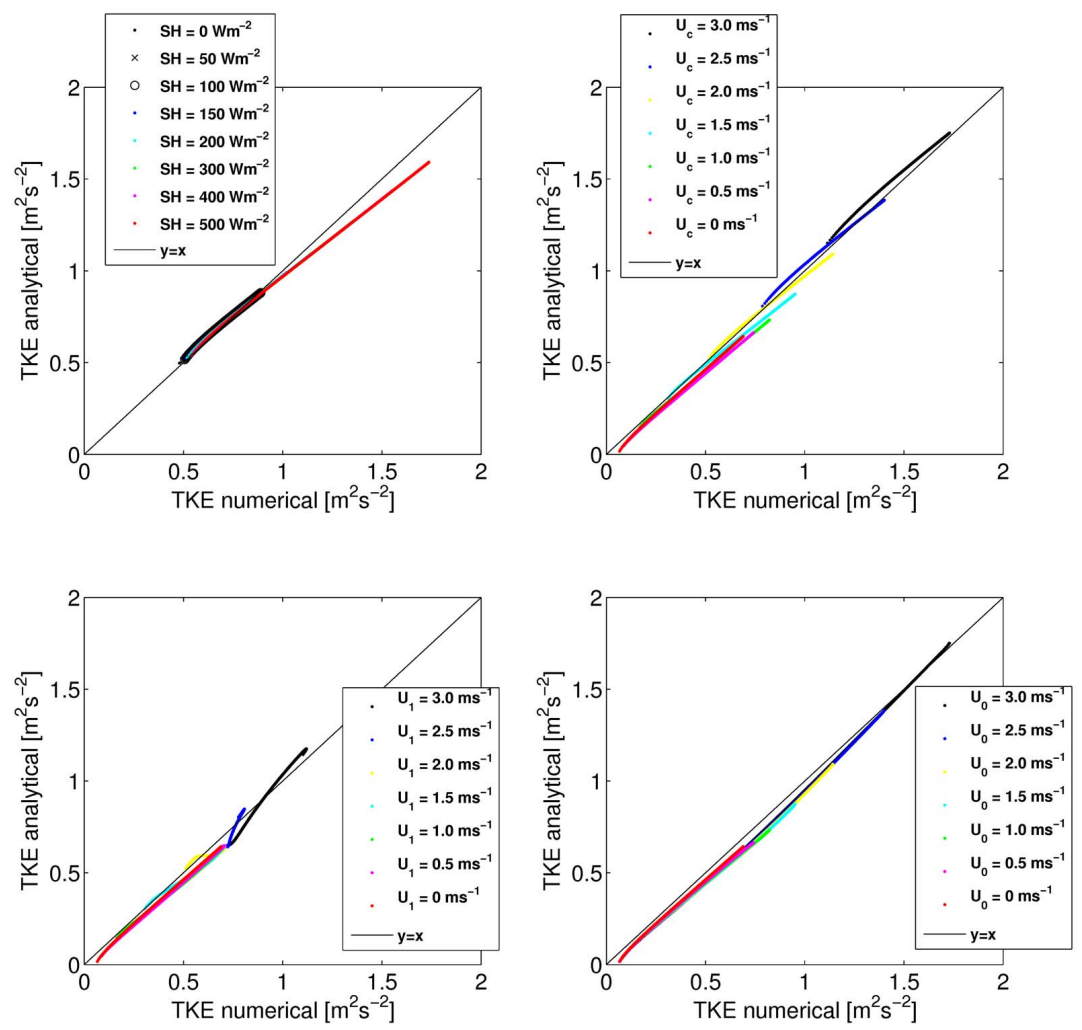

Figure 13. The figure shows a comparison between the numerically modeled TKE and the simplified analytical quasi-stationary expression of Eq. (20). The numerical model simulations correspond to those shown in Fig. 12, with SH runs shown in the upper left, $U_{\mathrm{c}}$ runs in the upper right, $U_{\mathrm{inc}}$ in the lower left and $U_{\mathrm{dec}}$ runs in the lower right. Each simulation is identified in the legend of each panel by its set variable value.

can even increase or stay more or less constant. These types of situations emphasize the limitations of simple exponent decay laws.

\subsection{A simple equilibrium model}

To better understand our numerical model results for TKE, near the surface we can compare our numerical results to a simple analytical expression for TKE which comes from assuming quasi-stationarity such that $\frac{\mathrm{d} E}{\mathrm{~d} t} \approx 0$ in Eq. (1). Additionally, we simplify the transport fraction $T_{\mathrm{f}}$ as 0.4 , ignoring the weak dependence on atmospheric stratification such that about 0.6 times the total near-surface production is balanced by local dissipation. We use Eqs. (3) and (4) together with the definition of friction velocity $\left(u_{*}^{2}=-\overline{u^{\prime} w^{\prime}}\right)$ for the shear production term, and the definition of convective velocity scale $\left(w_{*}^{3}=\frac{g z_{i}}{\theta_{\mathrm{v}}}\left(\overline{w^{\prime} \theta_{\mathrm{v}}^{\prime}}\right)_{\mathrm{S}}\right)$ for the buoyancy production term. Then, rearranging the TKE budget terms and solving for TKE from our dissipation parametrization (Eq. 16) yields

$$
\begin{aligned}
E^{3 / 2}= & \frac{0.6 l_{\epsilon} u_{*}^{3}}{k z}\left(1+3.6 k^{2 / 3}\left(\frac{z}{z_{i}}\right)^{2 / 3}\left(\frac{w_{*}}{u_{*}}\right)^{2}\right)^{-1 / 2}+ \\
& +\frac{0.6 l_{\epsilon} w_{*}^{3}}{z_{i}} .
\end{aligned}
$$

Here, $z / L$ in the wind gradient expression has also been rewritten in terms of $w_{*}$ and $u_{*}$ using $\frac{z}{L}=\frac{-k z w_{*}^{3}}{z_{i} u_{*}^{3}}$ (Stull, 1988 ) as it may be more instructive to consider how TKE in this simplified analytical expression is influenced by these governing velocity scales. This equation becomes a function of $u_{*}, z$ and $z_{i}$ when $w_{*}$ is small and conversely a function of $w_{*}, z$ and $z_{i}$ when $u_{*}$ is small, which seems reasonable. The influence of $z_{i}$ on near-surface TKE may be questionable, but it is consistent with our findings from Part 1 and $z_{i}$ used by Nadeau et al. (2011). A comparison is shown in Fig. 13 between numerically modeled TKE and TKE determined from Eq. (20) for the simulation results shown in Fig. 12. It is clear that the simplified equation is within about $10 \%$ of the numerical model results for TKE (at worst and often better) at this near-surface height of $2 \mathrm{~m}$. This success of simplification and ignoring the time dependence for the very near-surface 

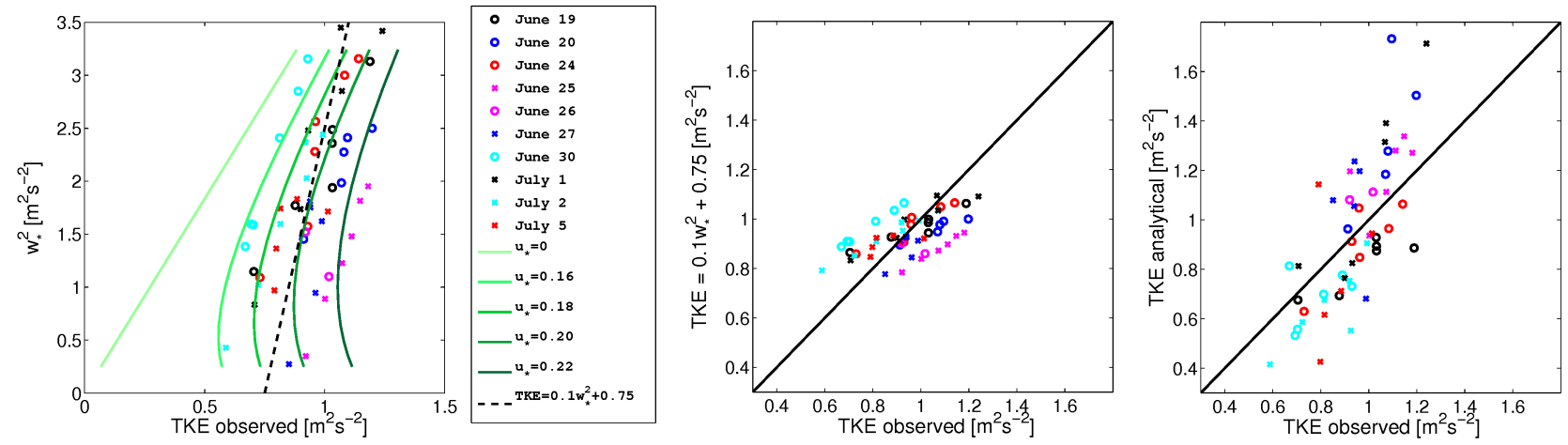

Figure 14. Left: observed $w_{*}^{2}$ as a function of observed TKE using hourly afternoon data. Here, Eq. (20) is illustrated with green colored lines for five different values of $u_{*}$ and $z_{i}=750 \mathrm{~m}$. A dashed black line is also shown as a fit to all the data. The hourly data have been assigned different colored symbols for different days as described in the legend and the same symbols are used in the middle and right panels. Middle: TKE calculated from the simple linear fit plotted as a function of the observed TKE. Right panel: TKE estimated from Eq. (20) plotted against observed TKE. Here, the observed $u_{*}, z_{i}$ and $w_{*}$ values have been used together with Eq. (20).

TKE is interesting, but we should bear that in mind by using prescribed functions of $z$ for each term. We thereby force all levels of the boundary layer to respond together. It is possible that we therefore underestimate the role of time dependence in the real boundary layer.

For a convective boundary layer with little shear production our expression reduces to $E=w_{*}^{2}\left(\frac{0.6 l_{\epsilon}}{z_{i}}\right)^{2 / 3}$, which gives a relatively low TKE in the surface layer of about $0.175 w_{*}^{2}$ and $0.413 w_{*}^{2}$, assuming a $1000 \mathrm{~m}$ boundary layer depth. This is low in comparison to earlier studies; for example, Caughey and Palmer (1979) give expressions for about $0.359 w_{*}^{2}$ and $0.544 w_{*}^{2}$. Our model can, however, also give some higher TKE when shear production is present.

In Fig. 14 (left panel), the simplified analytical expression is used to illustrate the dependence of TKE on $w_{*}$ for five different values of $u_{*}$ (green colored lines) and a fixed boundary layer depth of $750 \mathrm{~m}$. The plot also shows hourly data from the afternoon period with different colored symbols for the different days. The color scheme for the data is consistent with Part 1. That is, windier afternoons are shown in magenta, while the lowest wind speeds are shown in light blue. The results indicate that the simple expression for TKE variation is consistent with the observed trend of increasing TKE for increasing wind speed and $u_{*}$ and reverts back to a $w_{*^{-}}$ only expression for zero $u_{*}$. Included in the figure is also a simple linear fit between observed TKE and $w_{*}$ as a dashed black line ignoring the observed trend in $u_{*}$.

The middle panel of Fig. 14 shows TKE calculated by this simple linear fitted expression (TKE $=0.1 w_{*}^{2}+0.75$ ) using the observed $w_{*}$ compared to the observed TKE. This is done to provide a very simple reference model (or fit) to have something to compare with calculated evaluation metrics for Eq. (20). It is seen that the variability of calculated TKE from our simple linear fit is lower than in the observed data. For days with low wind the model overpredicts TKE, while for days with stronger winds the model underpredicts TKE.

The right panel of Fig. 14 shows TKE calculated from Eq. (20) and observed TKE. Here, we used the observed $z_{i}$ and $u_{*}$ in addition to the observed $w_{*}$ (as was also done for the simple linear fitted model). The figure shows that the variability of predicted TKE is larger than the observed TKE. Evidently, sometimes $u_{*}$ observations have large hourly variability, which is not always linked to a large increase or decrease in TKE. For example, on 20 June (Fig. 8) there is $1 \mathrm{~h}$ with observed $u_{*}>0.3$ that is significantly higher than the surrounding hours when $u_{*} \approx 0.22$. This is the hour that leads to the highest predicted TKE value when using Eq. (20) and has the largest individual hourly error. It is also possible that the analytical model is somewhat overly sensitive to some of the input parameters, contributing to variability in TKE that exceeds the observations. Windier afternoons appear to be associated with an overprediction of TKE, while those with very weak winds underpredict TKE somewhat. These trends, however, are not as clear as for the simple linear-fit model which only takes $w_{*}$ into account.

It is not possible to conclude with the evaluation metrics used here whether the TKE prediction was improved by including $u_{*}$ compared to an expression that only uses $w_{*}$. However, since Eq. (20) is based on relevant scaling variables, we consider it preferable to the empirical linear fit. The model presented here is also consistent with the observed TKE budget at the divergence site during the BLLAST field campaign, but should obviously be further tested on other sites and data before any conclusion on general validity can be made. 


\section{Discussion and conclusions}

This study presents a simple one-dimensional model to investigate atmospheric turbulence kinetic energy in sheared convective boundary layers. Similar to a previously proposed heuristic model for the surface layer TKE decay (Nadeau et al., 2011), our model uses boundary layer depth and buoyancy flux as input variables. However, we also include nearsurface wind speed to handle less convectively forced situations. The model is based on a simplified TKE budget including idealized height-varying terms for shear production, buoyant production, transport and dissipation, which together with initial conditions provides a basis for the evolution of TKE during the unstable part of the day until the start of stable conditions.

In the present work, the model was first run constrained by observations from the BLLAST field campaign for nine IOP days with relatively successful results for a near-surface TKE observed at $2.23 \mathrm{~m}$. Further above the ground, results were not as good, but still quite reasonable (illustrated by measurements at $61.4 \mathrm{~m}$ ). The height dependence of each budget term and therefore TKE can be challenging to model correctly. This was illustrated by the shear production term being rather well predicted near the surface, but its performance degraded with height. This height dependence of shear production proved difficult to infer from a single height wind measurement and further work is required to improve upon this model deficiency. Also, the model is unable to capture all the hourly variability observed in TKE budget terms and TKE. As long as no large systematic errors occur over extended periods of time, the mean TKE level is, however, quite well predicted by the model in many situations for these 9 days. As discussed in Part 1, the nine studied days include a variety of atmospheric stability conditions. The fact that the model is able to simulate roughly the right magnitude and temporal variations in near-surface TKE, both on more convective days and in more sheared conditions (e.g., 26 June), suggests that the budget relationships presented in Part 1 are realistic and can be used for atmospheric modeling applications and explorations.

The simple TKE model with all its discussed deficiencies still often yields quite realistic predictions of the overall evolution of boundary layer TKE and dissipation rate throughout the ABL depth. Further evaluation and investigation into the differences between the model and observations (as well as differences between different instrument estimates) may be needed. Currently, the model tends to predict quite high TKE dissipation rates when forced by observations from a forest site and wheat field. Also, using a $2 \mathrm{~km} \times 2 \mathrm{~km}$ areaaveraged flux gives modeled TKE dissipation rates which are high compared to observations. This is probably partly due to modeling uncertainties of urban and bare-soil conditions where no measurements were available. Observed dissipation rates are often lower than in the model. Elevated shear and the effects of different entrainment rates on different days may also be very important.

The model was used to illustrate its usefulness in understanding afternoon transition physics. The simple model was used to identify a region of reduced turbulence that starts in the upper parts of the boundary layer (but below the capping inversion), which moves down with time toward the surface. This phenomenon was conceptually described as a pre-residual layer. The pre-residual layer is consistent with the conclusions from Darbieu et al. (2015b), who noted that changes occur first in the upper part of the boundary layer during the later stages of the afternoon transition. In the preresidual layer, there is evidence that turbulence characteristics change as the layer forms with weak turbulence aloft, while the surface is still unstable. The process occurring during this time period influence the onset conditions for the actual residual layer, which resides entirely above the stable boundary layer. Further work should attempt to better understand the role of the pre-residual layer.

The model was further used in an idealized setting to illustrate the effects of relative amounts of shear and buoyancy for near-surface TKE at a height of $2 \mathrm{~m}$. It was illustrated that many different decay rates can exist in the afternoon. TKE that was both faster and slower than linearly decaying was possible when the model was driven with a simple timevarying wind. For a linearly increasing wind speed throughout the afternoon, TKE may increase during the afternoon despite a decrease in buoyancy production during the afternoon depending on shear production levels. This was also observed on 27 June, as discussed in Part 1. We also found that a linear decrease in TKE during an afternoon leads to a decay exponent value that is time-dependent and that no unique scaling law exponent exists in this situation.

We simplified our numerical model results to an analytical expression for quasi-stationary near-surface turbulence in Eq. (20). It compared well with the numerical results and was shown to reduce to an expression involving only $w_{*}$ as the relevant velocity scale in the case of zero friction velocity, and conversely to an expression involving only $u_{*}$ as the relevant velocity scale in the case of zero $w_{*}$. Both these cases are somewhat irrelevant for the atmospheric case in general because there will usually be some small amount of shear and buoyancy present. The fact that our numerical and analytical results compared relatively well indicates the usefulness of the quasi-stationarity assumption when describing the observed slow trends in the evolution of near-surface TKE. Further studies are, however, needed about the more rapid variations in TKE that occur on shorter timescales when forcing timescales also become comparable to large-eddy turnover timescales (van Driel and Jonker, 2011). In future work we should also confront our model with an LES model that uses the same measured fluxes as lower boundary conditions.

In reality, atmospheric turbulence kinetic energy is governed by many parameters, some of which have been included in the presented numerical model for TKE and others, 
such as horizontal advection and elevated wind shear, which remain to be included. In addition, TKE also has some memory of the history of the flow that we neglect when using Eq. (20). It should therefore be used with caution for prediction of turbulence kinetic energy. There is also no reason to be certain that, for instance, the near-surface transport fraction of about 0.4 determined from our measurements needs to apply to other data sets. However, we argue that the methodology from which our model originates is reasonably general and may also be attempted in future work on other sites and under other conditions.

\section{Data availability}

Metadata and data from the BLLAST campaign are available after registration at: http://bllast.sedoo.fr/. Processed data including TKE budget terms and model code is also available from the first-author upon request. 
Appendix A: Summary of model of height variation in TKE budget from Lenschow (1974)

For a qualitative comparison to our proposed height variation in TKE budget terms we provide here the simple model from Lenschow (1974). They discuss a turbulence kinetic energy equation normalized by buoyancy flux, and express it as

$H+T_{r}+S-D=0$

where $H$ and $S$ are the buoyancy- and shear-generation terms, $T_{r}$ the divergence of the vertical transport of turbulence energy and pressure fluctuations, and $D$ the dissipation rate.

Their expression for buoyancy production is given by

$H=1-1.15\left(z / z_{i}\right)$

for $z / z_{i} \leq 0.87$, and for $0.87 \leq z / z_{i} \leq 1$ they instead use

$H=-13.81+49.96(z / z i)-58.78\left(z / z_{i}\right)^{2}+22.53\left(z / z_{i}\right)^{3}$.

For shear production they use

$S=-\frac{L}{z_{i}}\left(1-15 \frac{z i}{L} \frac{z}{z i}\right)^{-1 / 4}$.

For dissipation rate their expression is

$D=0.43+\frac{0.57}{\langle S\rangle+3.75}(\langle S\rangle-S)+S$,

where $\langle S\rangle$ is an integrated shear production over the boundary layer depth given by $\langle S\rangle=-\frac{L}{z_{i}}\left[\ln \frac{z}{z_{0}}-\psi_{1}(x)\right]$, under the assumption of a constant shear stress throughout the surface and mixed layer. Here $x=\left(1-15 z_{i} / L\right)^{1 / 4}$, and $\psi_{1}(x)=$ $2 \ln \frac{1+x}{2}+\ln \frac{1+x^{2}}{2}-2 \tan ^{-1} x+\pi / 2$.

In addition, for transport they use

$T r=0.43+\frac{0.57}{\langle S\rangle+3.75}(\langle S\rangle-S)-H$

This model from Lenschow (1974) is illustrated (in black) for a more convective case $\left(z_{i} / L=-1000\right)$ in Fig. A1 and for a more neutral case $\left(z_{i} / L=-1\right)$ in Fig. A2. Also included are our modeled vertical profiles of TKE budget terms (in red) from 2 July at times with the same overall stratification. The TKE tendency term is also included as blue lines. See Sect. 2.4 for discussions concerning the vertical profiles of TKE budget terms in these two models. 

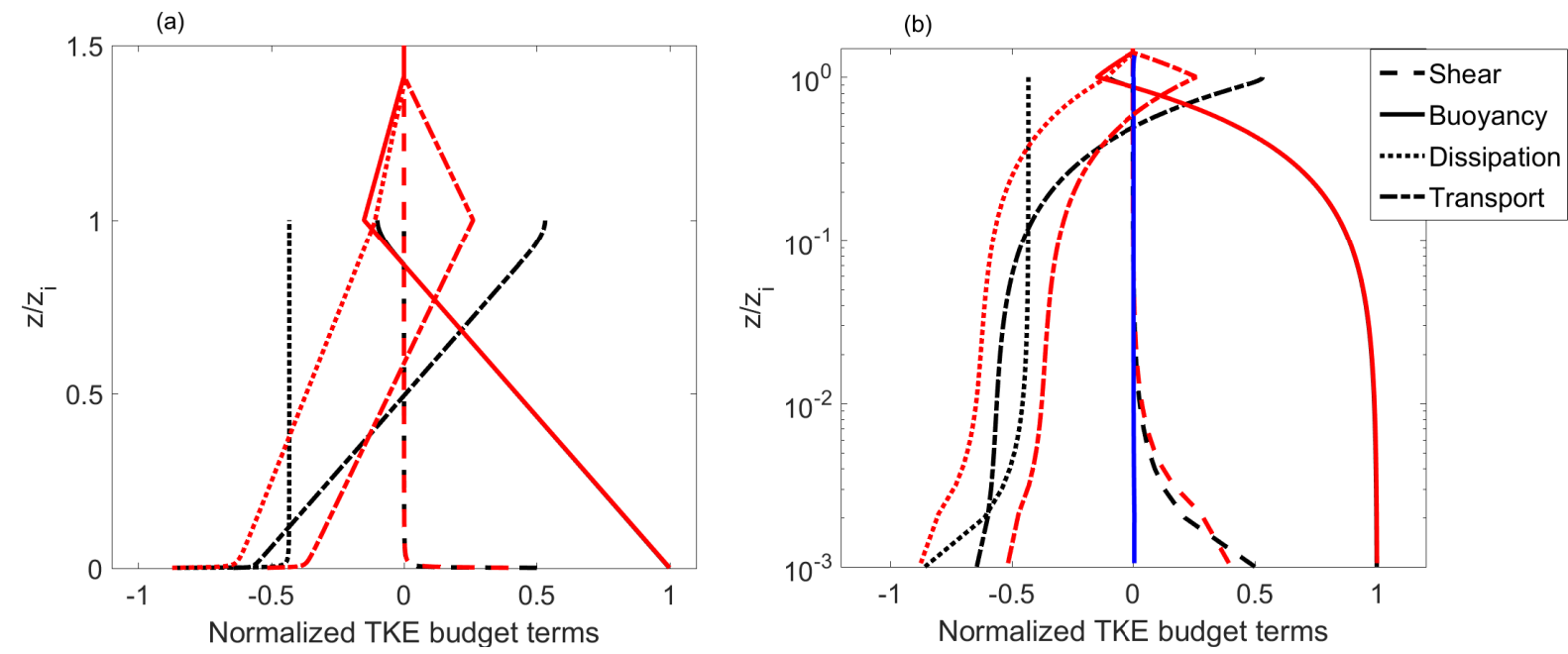

Figure A1. Normalized terms in the turbulence kinetic energy equation from Lenschow (1974) (in black) and from our model (in red) for a convective case with $z_{i} / L$ equal to $-1000, z_{i} \approx 1000 \mathrm{~m}$ and a surface roughness length $z_{0}=0.02$ m. Buoyancy production, shear production, dissipation and transport are shown with full, dashed, dotted and dash-dotted lines, respectively, for normalized height $z / z_{i}$ in panel (a) and with a logarithmic $y$ axis in panel (b). The TKE tendency term in our model is shown in blue in panel (b).

(a)

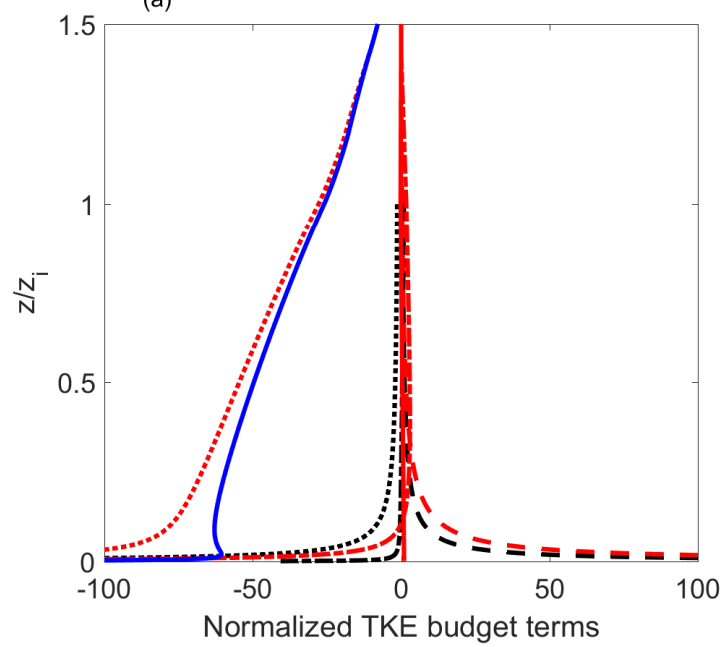

(b)

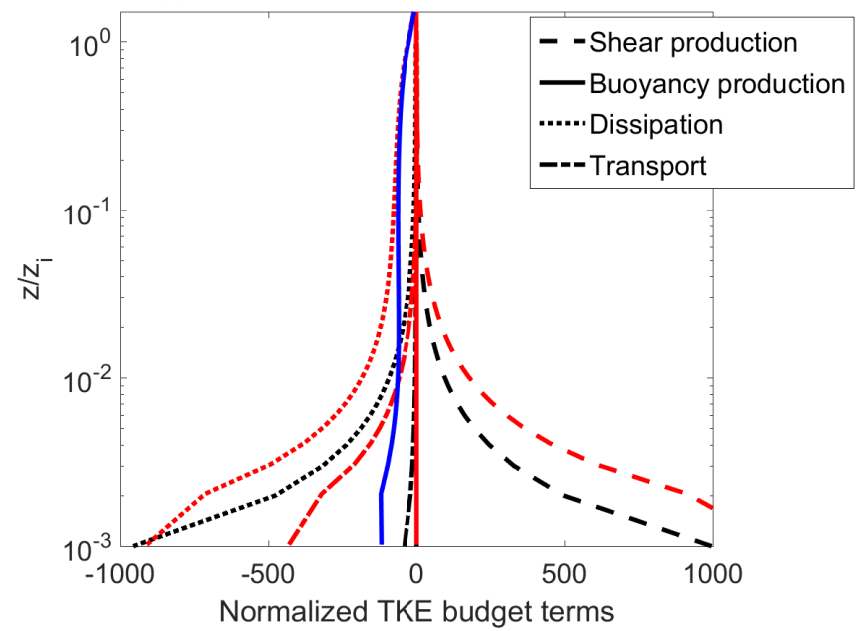

Figure A2. Normalized terms in the turbulence kinetic energy equation from Lenschow (1974) (in black) and our model (in red) for a very near neutral case with $z_{i} / L$ equal to $-1, z_{i} \approx 1000 \mathrm{~m}$ and a surface roughness length $z_{0}=0.02 \mathrm{~m}$. Buoyancy production, shear production, dissipation and transport are shown with full, dashed, dotted and dash-dotted lines, respectively, for normalized height $z / z_{i}$ in panel (a) and with a logarithmic $y$ axis in panel (b). The TKE tendency term in our model is shown in blue. 
Acknowledgements. The first author thanks ANR for funding this postdoctoral work and would also like to thank Jordi Vilà-Guerau de Arellano and Arnold Moene at Wageningen University for fruitful discussions about this work during a research visit in December 2014. The BLLAST field experiment was made possible thanks to the contribution of several institutions and sources of support: INSU-CNRS (Institut National des Sciences de l'Univers, Centre national de la Recherche Scientifique, LEFE-IMAGO program), Météo-France, Observatoire Midi-Pyrénées (University of Toulouse), EUFAR (EUropean Facility for Airborne Research) BLLATE-1\&2, and COST ES0802 (European Cooperation in Science and Technology). The field experiment would not have occurred without the contribution of all participating European and American research groups, which all have contributed to a significant extent. The BLLAST field experiment was hosted by the instrumented site of Centre de Recherches Atmosphériques, Lannemezan, France (Observatoire Midi-Pyrénées, Laboratoire d'Aérologie). Its $60 \mathrm{~m}$ tower is partly supported by the POCTEFA/FLUXPYR European program. The authors also thank Guylaine Canut, Yannick Bezombes, Solène Derrien, Bernard Campistron and Frédérique Saïd for their involvement in the measurements used here. Fleur Couvreux and Patrick Augustin are acknowledged for their contribution to the estimates of the $\mathrm{ABL}$ depth and Pierre Durand for his estimates of dissipation rate from aircraft. BLLAST data are managed by SEDOO, from Observatoire Midi-Pyrénées. See http://bllast.sedoo.fr for all contributions. Since 2013, the French ANR has supported BLLAST analysis. This research was also partially funded by the Office of Naval Research (award no. N00014-11-1-0709) and the Mountain Terrain Atmospheric Modeling and Observations (MATERHORN) program. The authors are grateful to Daniel Alexander for his technical efforts in making measurements at the forest site and divergence site possible.

Edited by: Robert John Beare

Reviewed by: two anonymous referees

\section{References}

Angevine, W., Baltink, H., and Bosveld, F.: Observations Of The Morning Transition Of The Convective Boundary Layer, Bound.Lay. Meteorol., 101, 209-227, 2001.

Basu, S., Vinuesa, J., and Swift, A.: Dynamic LES modeling of a diurnal cycle, J. Appl. Meteorol. Climatol., 47, 1156-1174, 2008.

Beare, R. J., Edwards, J. M., and Lapworth, A.: Simulation of the observed evening transition and nocturnal boundary layers: large-eddy simulation, Q. J. Roy. Meteor. Soc., 132, 81-99, 2006.

Blay-Carreras, E., Pino, D., Vilà-Guerau de Arellano, J., van de Boer, A., De Coster, O., Darbieu, C., Hartogensis, O., Lohou, F., Lothon, M., and Pietersen, H.: Role of the residual layer and large-scale subsidence on the development and evolution of the convective boundary layer, Atmos. Chem. Phys., 14, 4515-4530, doi:10.5194/acp-14-4515-2014, 2014.

BLLAST: Boundary Layer Late Afternoon and Sunset Turbulence (BLLAST) website, available at: http://bllast.sedoo.fr/database/ (last access: 1 July 2016), 2015.

Brown, A., Cederwall, R., Chlond, A., Duynkerke, P., Golaz, J., Khairoutdinov, M., Lewellen, D., Lock, A., MacVean, M., Moeng, C.-H., R. A. J., N., Siebsma, A., and Stevens, B.: Large-eddy simulation of the diurnal cycle of shallow cumulus convection over land, Q. J. Roy. Meteor. Soc., 128, 1075-1093, 2002.

Canut, G., Couvreux, F., Lothon, M., Legain, D., Piguet, B., Lampert, A., and Moulin, E.: Turbulence measurements with a tethered balloon, Atmos. Meas. Tech. Discuss., doi:10.5194/amt2015-386, in review, 2016.

Caughey, S. and Palmer, S.: Some aspects of turbulence structure through the depth of the convective boundary layer, Q. J. Roy. Meteor. Soc., 105, 811-827, 1979.

Couvreux, F., Bazile, E., Canut, G., Seity, Y., Lothon, M., Lohou, F., Guichard, F., and Nilsson, E.: Boundary-layer turbulent processes and mesoscale variability represented by Numerical Weather Prediction models during the BLLAST campaign, Atmos. Chem. Phys. Discuss., doi:10.5194/acp-2015-1042, in review, 2016.

Darbieu, C., Lohou, F., Lothon, M., de Arellano, J. V.-G., Couvreux, F., Durand, P., Pino, D., Patton, E. G., Nilsson, E., Blay-Carreras, E., and Gioli, B.: Interactive comment on "Turbulence vertical structure of the boundary layer during the afternoon transition" by C. Darbieu et al., http://www.atmos-chem-phys-discuss.net/14/C13233/2015/ acpd-14-C13233-2015-supplement.pdf, Atmos. Chem. Phys. Discuss., 14, C13233-C13233, 2015 a.

Darbieu, C., Lohou, F., Lothon, M., Vilà-Guerau de Arellano, J., Couvreux, F., Durand, P., Pino, D., Patton, E. G., Nilsson, E., Blay-Carreras, E., and Gioli, B.: Turbulence vertical structure of the boundary layer during the afternoon transition, Atmos. Chem. Phys., 15, 10071-10086, doi:10.5194/acp-1510071-2015, 2015. b.

Deardorff, J.: Numerical investigation of neutral and unstable planetary boundary layers, J. Atmos. Sci., 29, 91-115, 1972.

De Coster, O. and Pietersen, H.: BLLAST - uniform processing of Eddy-Covariance data, available at: http: //bllast.sedoo.fr/documents/reports/H-Pietersen_O-de-Coster_ BLLAST-surf_flx-uniform-processing.pdf (last access: 1 July 2016), 2012.

Dupuis H., Taylor, P. K., Weill, A., and Katsaros, K.: Inertial dissipation method applied to derive turbulent fluxes over the ocean during the Surface of the Ocean, Fluxes and Interactions with the Atmosphere/Atlantic Stratocumulus Transition Experiment (SOFIA/ASTEX) and Structure des Echanges Mer-Atmosphere, Proprietes des Heterogeneites Oceaniques: Recherche Experimentale (SEMAPHORE) experiments with low to moderate wind speeds, J. Geophys. Res., 102, 21115-21129, 1997.

Garcia, D.: Robust smoothing of gridded data in one and higher dimensions with missing values, Comput. Stat. Data Anal., 54, 1167-1178, 2010.

Garratt, J.: The Atmospheric Boundary Layer, Cambridge University Press, 316 pp, 1992.

Goulart, A., Degrazia, G., Rizza, U., and Anfossi, D.: A theoretical model for the study of convective turbulence decay and comparison with large-eddy simulation data, Bound.-Lay. Meteorol., 107, 143-155, 2003.

Goulart, A., Bodmann, B., de Vilhena, M., Soares, P., and Moreira, D.: On the Time Evolution of the Turbulent Kinetic Energy Spectrum for Decaying Turbulence in the Convective Boundary Layer, Bounda.-Lay. Meteorol., 138, 61-75, 2010. 
Grimsdell, A. W. and Angevine, W. M.: Observations of the afternoon transition of the convective boundary layer, J. Appl. Meteor., 41, 3-11, 2002.

Hartogensis, O. K.: BLLAST Flux maps, available at: http://bllast.sedoo.fr/workshops/february2015/presentations/ Hartogensis-Oscar_area-averaged-flux.pdf (last access: 1 July 2016), 2015.

Högström, U.: Non-dimensional wind and temperature profiles in the atmospheric surface layer, Bound.-Lay. Meteorol., 42, 263270,1988

Jacoby-Koaly, S., Campistron, B., Bernard, S., Bönech, B., Ardhuin-Girard, F., Dessens, J., Dupont, E., and Carissimo, B.: Turbulent Dissipation Rate in the Boundary Layer via UHF Wind Profiler Doppler Spectral Width Measurements, Bound.Lay. Meteorol., 103, 361-389, 2002.

Kumar, V., Kleissl, J., Meneveau, C., and Parlange, M.: Large eddy simulation of a diurnal cycle of the atmospheric boundary layer: Atmospheric stability and scaling issues, Water Resour. Res., 42, $3-18,2006$.

Lenschow, D., Wyngaard, J., and Pennel, W.: Mean-field and second-moment budgets in a baroclinic, convective boundary layer, J. Atmos. Sci., 37, 1313-1326, 1980.

Lenschow, D. H.: Model of Height Variation of the Turbulence Kinetic Energy Budget in the Unstable Planetary Boundary Layer, J. Atmos. Sci., 31, 465-474, 1974.

Lothon, M., Lohou, F., Pino, D., Couvreux, F., Pardyjak, E. R., Reuder, J., Vilà-Guerau de Arellano, J., Durand, P., Hartogensis, O., Legain, D., Augustin, P., Gioli, B., Lenschow, D. H., Faloona, I., Yagüe, C., Alexander, D. C., Angevine, W. M., Bargain, E., Barrié, J., Bazile, E., Bezombes, Y., Blay-Carreras, E., van de Boer, A., Boichard, J. L., Bourdon, A., Butet, A., Campistron, B., de Coster, O., Cuxart, J., Dabas, A., Darbieu, C., Deboudt, K., Delbarre, H., Derrien, S., Flament, P., Fourmentin, M., Garai, A., Gibert, F., Graf, A., Groebner, J., Guichard, F., Jiménez, M. A., Jonassen, M., van den Kroonenberg, A., Magliulo, V., Martin, S., Martinez, D., Mastrorillo, L., Moene, A. F., Molinos, F., Moulin, E., Pietersen, H. P., Piguet, B., Pique, E., RománCascón, C., Rufin-Soler, C., Saïd, F., Sastre-Marugán, M., Seity, Y., Steeneveld, G. J., Toscano, P., Traullé, O., Tzanos, D., Wacker, S., Wildmann, N., and Zaldei, A.: The BLLAST field experiment: Boundary-Layer Late Afternoon and Sunset Turbulence, Atmos. Chem. Phys., 14, 10931-10960, doi:10.5194/acp14-10931-2014, 2014.

Mangia, C., Degrazia, G. A., and Rizza, U.: An Integral Formulation for the Dispersion Parameters in a Shear-Buoyancy-Driven Planetary Boundary Layer for Use in a Gaussian Model for Tall Stacks, J. Appl. Meteorol., 39, 1913-1922, 2000.

Moeng, C.-H. and Sullivan, P. P.: A comparison of shear and buoyancy driven planetary-boundary-layer flows, J. Atmos. Sci., 51, 999-1022, 1994.

Nadeau, D. F., Pardyjak, E. R., Higgins, C. W., Fernando, H. J. S., and Parlange, M. B.: A simple model for the afternoon and early evening decay of convective turbulence over different land surfaces, Bound.-Lay. Meteorol., 141, 301-324, 2011.
Nieuwstadt, F. T. M. and Brost, R. A.: The decay of convective turbulence, J. Atmos. Sci., 43, 532-546, 1986.

Nilsson, E., Lohou, F., Lothon, M., Pardyjak, E., Mahrt, L., and Darbieu, C.: Turbulence kinetic energy budget during the afternoon transition - Part 1: Observed surface TKE budget and boundary layer description for 10 intensive observation period days, Atmos. Chem. Phys., 16, 8849-8872, doi:10.5194/acp-16-88492016, 2016.

Pietersen, H. P., Vilà-Guerau de Arellano, J., Augustin, P., van de Boer, A., de Coster, O., Delbarre, H., Durand, P., Fourmentin, M., Gioli, B., Hartogensis, O., Lohou, F., Lothon, M., Ouwersloot, H. G., Pino, D., and Reuder, J.: Study of a prototypical convective boundary layer observed during BLLAST: contributions by large-scale forcings, Atmos. Chem. Phys., 15, 42414257, doi:10.5194/acp-15-4241-2015, 2015.

Pino, D., Jonker, H. J. J., Vilà de Arellano, J., and Dosio, A.: Role of shear and the inversion strength during sunset turbulence over land: characteristic length scales, Bound.-Lay. Meteorol., 121, 537-556, 2006.

Puhales, F., Rizza, U., Degrazia, G., and Acevedo, O.: A simple parameterization for the turbulent kinetic energy transport terms in the convective boundary layer derived from large eddy simulation, Physica A, 392, 583-595, 2013.

Rizza, U., Miglietta, M., Degrazia, G., Acevedo, O., and Marques, E.: Sunset decay of the convective turbulence with Large-Eddy Simulation under realistic conditions, Physica A, 392, 44814490, 2013.

Sorbjan, Z.: Decay of convective turbulence revisited, Bound.-Lay. Meteorol., 82, 501-515, 1997.

Stull, R. B.: An introduction to boundary layer meteorology, Kluwer Academic Publishers, 666 pp., 1988.

Van Dijk, A., Moene, A., and De Bruin, H.: The principles of surface flux physics: theory, practice and description of the ECPACK-library, Tech. rep., 2004.

van Driel, R. and Jonker, H. J. J.: Convective Boundary Layers Driven by Nonstationary Surface Heat Fluxes, J. Atmos. Sci., 68, 727-738, 2011.

Verkaik, J. and Holtslag, A. A. M.: Wind profiles, momentum fluxes and roughness lengths at Cabauw revisited, Bound.-Lay. Meteorol., 122, 701-719, 2007.

Wilson, K.: An alternative function for the wind and temperature gradients in unstable surface layers, Bound.-Lay. Meteorol., 99, 151-158, 2001.

Wingo, S. M. and Knupp, K. R.: Multi-Platform Observations Characterizing the Afternoon-to-Evening Transition of the Planetary Boundary Layer in Northern Alabama, USA, Bound.-Lay. Meteorol., 155, 29-53, 2015.

Wyngaard, J. C.: Turbulence in the Atmosphere, Cambridge University Press, New York, 393 pp., 2010. 